\title{
Loop structures in Taylor towers
}

\author{
GREGORY Z ARONE \\ WILLIAM G DWYER \\ KATHRYN LESH
}

\begin{abstract}
We study spaces of natural transformations between homogeneous functors in Goodwillie's calculus of homotopy functors and in Weiss's orthogonal calculus. We give a description of such spaces of natural transformations in terms of the homotopy fixed point construction. Our main application uses this description in combination with the Segal Conjecture to obtain a delooping theorem for connecting maps in the Goodwillie tower of the identity and in the Weiss tower of $B U(V)$. The interest in such deloopings stems from conjectures made by the first and the third author [4] that these towers provide a source of contracting homotopies for certain projective chain complexes of spectra.
\end{abstract}

55P65; 55P47, 18G55

\section{Introduction and notation}

In this paper, we study homogeneous functors in the sense of Weiss's orthogonal calculus [13]. More precisely, we calculate the space of natural transformations between such functors, and we give a few examples and applications. Our main application is a delooping theorem for connecting maps in the Goodwillie tower of the identity functor for pointed spaces and in the Weiss tower of the functor $V \mapsto B U(V)$. The motivation for this delooping theorem will be discussed later in this introduction.

To state our results, we summarize the usual notation for this context. Let $F$ be a functor from pointed spaces or from finite-dimensional real or complex vector spaces to pointed spaces or spectra. Goodwillie and Weiss calculus assign to such a functor $F$ a "Taylor" tower of functors

$$
\cdots \rightarrow \mathrm{P}_{n} F \rightarrow \mathrm{P}_{n-1} F \rightarrow \cdots,
$$

together with a natural map from $F$ to the homotopy inverse limit of the tower that is often a weak homotopy equivalence. The homotopy fiber of the map $\mathrm{P}_{n} F \rightarrow \mathrm{P}_{n-1} F$ is customarily denoted $\mathrm{D}_{n} F$ and is referred to as "the $n$th homogeneous layer of $F$," while $\mathrm{P}_{n} F$ is referred to as "the $n$th Taylor polynomial of $F$." Depending on whether 
the source category is pointed spaces (Goodwillie calculus, or "homotopy calculus") or finite-dimensional complex vector spaces (Weiss calculus, or "orthogonal calculus"), the $n$th homogeneous layer is naturally equivalent to a functor of one of the following forms:

$$
\begin{aligned}
& X \mapsto \Omega^{\infty}\left(C \wedge \Sigma^{\infty} X^{\wedge n}\right)_{\tilde{h} \Sigma_{n}} \\
& V \mapsto \Omega^{\infty}\left(C \wedge \Sigma^{\infty} S^{n V}\right)_{\tilde{h} U(n)}
\end{aligned}
$$

Here $n V$ is the vector space $\mathbb{C}^{n} \otimes V$, the space $S^{n V}$ is the one-point compactification of $n V$, and $C$ is a spectrum with an action of the symmetric group $\Sigma_{n}$ or of the unitary group $U(n)$, as appropriate. One calls $C$ the " $n$th derivative" of the functor $F$.

Let $F$ be a reduced functor in the context of either the homotopy calculus or the orthogonal calculus. The fibration sequence $\mathrm{D}_{n+1} F \rightarrow \mathrm{P}_{n+1} F \rightarrow \mathrm{P}_{n} F$ is induced from a natural transformation $\mathrm{P}_{n} F \rightarrow \mathrm{B} \mathrm{D}_{n+1} F$, and we call the composite

$$
\mathrm{D}_{n} F \rightarrow \mathrm{P}_{n} F \rightarrow \mathrm{B} \mathrm{D}_{n+1} F
$$

the " $n$th connecting map" or the " $n$th structure map" in the Taylor tower. (If $F$ is not reduced, the connecting map can still be defined as the connecting map of the reduction of $F$.)

In this paper, we prove delooping results for certain connecting maps by studying spaces of homotopy natural transformations between homogeneous functors. To prepare the foundation for our calculations, we give a careful construction of a space hoNat $(F, G)$ of homotopy natural transformations from a functor $F$ to a functor $G$. The set of path components of hoNat $(F, G)$ can be identified with the set of morphisms from $F$ to $G$ in the homotopy category of functors. It is constructed as a derived functor of the ordinary space of natural transformations, $\operatorname{Nat}(F, G)$, using standard techniques of Quillen model categories. This work is carried out in Section 2 and consists largely of formalizing ideas implicitly used in the work of Weiss [13].

The starting point for most of our delooping results is Theorem 3.2 below. Let $\mathcal{J}$ be the category of finite-dimensional complex vector spaces with inner product and innerproduct preserving linear maps. The theorem describes natural transformations between homogeneous functors on $\mathcal{J}$ in terms of mapping spaces involving the representing spectra $C$ and $D$ together with actions of their respective unitary groups. It is a typical calculation of its kind, very much like calculations done by Goodwillie from the inception of the calculus of functors; indeed, the result is more or less implicit in the work of Weiss [13]. However, we have tried to give the proof in more detail than has perhaps been done in the literature so far. 
Theorem 3.2 Let $C$ and $D$ be spectra with actions of $U(n)$ and $U(k)$, respectively. Then

$$
\begin{aligned}
\operatorname{hoNat}_{\mathcal{J}}\left[\left(C \wedge \Sigma^{\infty} S^{n V}\right)_{\tilde{h} U(n)},\left(D \wedge \Sigma^{\infty} S^{k V}\right)_{\tilde{h} U(k)}\right] & \\
& \left.\simeq \operatorname{Map}\left(C,\left(D \wedge \operatorname{mor}_{\mathcal{J}}\left(\mathbb{C}^{n}, \mathbb{C}^{k}\right)_{+}\right)_{\tilde{h} U(k)}\right)\right)^{\tilde{h} U(n)}
\end{aligned}
$$

Our main interest lies in examples. Our two main results (Theorems 6.1 and 7.1 below) involve finite deloopings, but we begin with the following theorem on infinite deloopings. This result is certainly known to Goodwillie, but we record it here with our foundational constructions as its basis.

Theorem 4.2 Let $F$ be a space-valued functor, to which either Goodwillie calculus or Weiss calculus applies. Suppose that there exists an $n$ such that the Taylor tower of $F$ has nontrivial layers only in degrees $n \leq i<2 n$. Then $F$ takes values in the category of infinite loop spaces.

In the case of the Goodwillie tower of the identity, Theorem 4.2 can be thought of as analogous to the fact if $\Gamma_{n} G$ is the $n$th term in the lower central series of $G$ then $\Gamma_{n} G / \Gamma_{2 n} G$ is an abelian group. The analogy comes because one can think of the Goodwillie tower of the identity as a homotopy analogue of the lower central series.

The main theorems of this paper are Theorems 6.1 and 7.1. The first is a delooping result for the structure maps in the Taylor tower of the identity functor, and the second is a similar result for the functor $V \mapsto B U(V)$ of finite-dimensional complex vector spaces.

To set the stage for Theorems 6.1 and 7.1, consider the identity functor on pointed spaces, or more precisely, the $p$-localization of this identity functor for a prime $p$. For the moment, let $P_{n}(X)$ and $D_{n}(X)$ denote the $n$th Taylor polynomial and the $n$th homogeneous layer of this functor. It is known from work of Arone and Mahowald [5] that if $X$ is an odd-dimensional sphere then $D_{n}(X) \simeq *$ unless $n=p^{k}$ for some $k$. (In the case $p=2$, the assumption that $X$ is odd-dimensional may be dropped.) Let $\mathrm{DI}_{k}(X)$ denote the $p^{k}$ th layer of the $p$-localized Taylor tower of the identity. It follows that when $X$ is an odd-dimensional sphere (any sphere, if $p=2$ ), there is a map in the homotopy category of spaces

$(1-1) \quad \kappa_{k}: \mathrm{DI}_{k}(X) \rightarrow P_{p^{k}}(X) \stackrel{\simeq}{\longleftarrow} P_{p^{k+1}-1}(X) \rightarrow \mathrm{BDI}_{k+1}(X)$. 
Note that the map $\kappa_{k}$ is well-defined only when $X$ is a sphere (odd-dimensional, if $p$ is odd). ${ }^{1}$ In particular, $\kappa_{k}$ is not a natural transformation of functors on the category of pointed spaces. To overcome this difficulty, we modify our point of view as follows: instead of thinking of spheres as the values of the identity functor from $\mathrm{Top}_{*}$ to $\mathrm{Top}_{*}$, we think of them as the values of the one-point compactification functor from vector spaces to Top ${ }_{*}$. More precisely, let $\mathcal{X}$ be the functor from complex vector spaces (resp. real vector spaces if $p=2$ ) defined by $\mathcal{X}(V)=\left(S^{1} \wedge S^{V}\right)_{(p)}$ (resp. $\mathcal{X}(V)=\left(S^{V}\right)_{(2)}$ if $\left.p=2\right)$, where the subscript ${ }_{(p)}$ denotes localization at $p$. General calculus considerations show that the Weiss tower of $\mathcal{X}$, evaluated at $V$, is the same as the Goodwillie tower of the identity, evaluated at $\mathcal{X}(V)$. Thus the chain of maps (1-1) may be reinterpreted as a chain of natural transformations of functors of vector spaces

$$
\mathrm{D}_{p^{k}} \mathcal{X}(V) \rightarrow \mathrm{P}_{p^{k}} \mathcal{X}(V) \stackrel{\simeq}{\longleftarrow} \mathrm{P}_{p^{k+1}-1} \mathcal{X}(V) \rightarrow \mathrm{B} \mathrm{D}_{p^{k+1}} \mathcal{X}(V) .
$$

Now the middle map is a weak equivalence for all objects $V$ in the domain category of $\mathcal{X}$. Thus the middle map is a weak equivalence of functors, and the composed map of (1-2) may be considered as a map in the homotopy category of functors. In other words it is, essentially, a natural transformation between homogeneous functors, and so it can be studied using Theorem 3.2. The following theorem implies that not only can this natural transformation be delooped, but in fact any natural transformation between these two functors can be delooped.

Theorem 6.1 Let $\mathcal{X}$ be the functor from finite-dimensional vector spaces over $\mathbb{C}$ (over $\mathbb{R}$ if $p=2$ ) given by $\mathcal{X}(V)=\left(S^{1} \wedge S^{V}\right)_{(p)}\left(\right.$ resp. $\mathcal{X}(V)=\left(S^{V}\right)_{(2)}$ if $\left.p=2\right)$. Then the $k$-fold looping map

$$
\Omega^{k}: \operatorname{hoNat}\left(\mathrm{B}^{k} \mathrm{D}_{p^{k}} \mathcal{X}, \mathrm{B}^{k+1} \mathrm{D}_{p^{k+1}} \mathcal{X}\right) \rightarrow \operatorname{hoNat}\left(\mathrm{D}_{p^{k}} \mathcal{X}, \mathrm{B} \mathrm{D}_{p^{k+1}} \mathcal{X}\right)
$$

is surjective on $\pi_{0}$.

The main ingredient in the proof is Theorem 6.3, which depends in an essential way on the Segal Conjecture.

There is a similar connecting map between the layers of the Weiss tower of the functor $V \mapsto B U(V)$, or more precisely, the $p$-completion of this functor. In this case, the connecting map can be thought of as a natural transformation without preliminary fiddling. We have the following companion theorem to Theorem 6.1, without the need for a separate case for $p=2$.

\footnotetext{
${ }^{1}$ When $X$ is not an appropriate sphere, the map $P_{p^{k}}(X) \leftarrow P_{p^{k+1}-1}(X)$ in the middle of $\kappa_{k}$ is not a weak equivalence.
} 
Theorem 7.1 Let $k>0$. Let $\mathrm{DU}_{k}(V)$ be the $p^{k}$ th layer of the $p$-completed Weiss tower of the functor $V \mapsto B U(V)$ from complex vector spaces to spaces. Then the $(k-1)$-fold looping map

$$
\Omega^{k-1}: \operatorname{hoNat}\left(\mathrm{B}^{k-1} \mathrm{DU}_{k}, \mathrm{~B}^{k} \mathrm{DU}_{k+1}\right) \rightarrow \operatorname{hoNat}\left(\mathrm{DU}_{k}, \mathrm{~B} \mathrm{DU}_{k+1}\right)
$$

is surjective on $\pi_{0}$.

As an immediate consequence of the theorems above, we obtain the deloopings for the specific Taylor towers that we actually wanted, as follows.

Corollary 6.2 Let $p$ be a prime and let $n=p^{k}$. Let $X$ be an odd-dimensional sphere (or any sphere, if $p=2$ ). Let $\operatorname{DI}_{k}(X)$ be the $p^{k}$ th layer of the $p$-localized Goodwillie tower of the identity evaluated at $X$. Then the connecting map

$$
\kappa_{k}: \mathrm{DI}_{k}(X) \rightarrow \mathrm{B} \mathrm{DI}_{k+1}(X)
$$

admits a $k$-fold delooping in the homotopy category of spaces.

Corollary 7.2 Let $p$ be a prime and let $n=p^{k}$ for $k>0$. Let $\operatorname{DU}_{k}(V)$ be the $p^{k}$ th layer of the $p$-completed Weiss tower of $B U(-)$ evaluated at a finite-dimensional complex vector space $V$. Then the connecting map

$$
\kappa_{k}: \mathrm{DU}_{k}(V) \rightarrow \mathrm{B} \mathrm{DU}_{k+1}(V)
$$

admits a $(k-1)$-fold delooping in the homotopy category of functors.

Remark 1.1 Theorem 7.1 and Corollary 7.2 require $p$-completion for technical reasons. The difference between these two statements and those of Theorem 6.1 and Corollary 6.2 is mostly illusory, since the layers of degree $>1$ in both of the towers in question have finite homotopy groups, and so for these layers $p$-completion is the same as $p$-localization. The Goodwillie tower for the odd sphere is rationally nontrivial in degree 1, while the Weiss tower for $B U(-)$ is rationally nontrivial in degrees 0 and 1 .

Before we say something about the proofs, we discuss the motivation for Corollaries 6.2 and 7.2. It has been believed since the writing of Arone and Mahowald [5] that there is a connection between the Goodwillie tower of the identity, evaluated at $S^{1}$, and the filtration of the Eilenberg-Mac Lane spectrum $\mathrm{H} \mathbb{Z}$ by the symmetric powers of sphere spectrum. This hypothetical picture was extended in Arone and Lesh [4], where the authors constructed a filtration of the connective $K$-theory spectrum $b u$ that is analogous to the symmetric power filtration and conjectured that there is a relationship between this new filtration and the Weiss tower of $B U(-)$. 
We explain the conjectured relationship in more detail. As before, let $\mathrm{DI}_{k}$ be the $p^{k}$ th layer of the $p$-localized Goodwillie tower of the identity evaluated at $S^{1}$, and let $\mathrm{DU}_{k}$ be the $p^{k}$ th layer of the $p$-completed Weiss tower of $B U(-)$ evaluated at $\mathbb{C}$. Let $\kappa_{k}$ be the $k$ th connecting map in either of these towers, as in Corollaries 6.2 and 7.2. Let

$$
f_{k}: \mathrm{B}^{k-1} \mathrm{DI}_{k} \longrightarrow \mathrm{B}^{k} \mathrm{DI}_{k+1}
$$

be a $(k-1)$-fold delooping of $\kappa_{k}$ in the homotopy case, ${ }^{2}$ and let

$$
g_{k}: \mathrm{B}^{k-1} \mathrm{DU}_{k} \longrightarrow \mathrm{B}^{k} \mathrm{DU}_{k+1}
$$

be a $(k-1)$-delooping in the unitary case. The existence of such deloopings is guaranteed by Corollaries 6.2 and 7.2. (In fact, in the homotopy case we are using one delooping less than is provided by the theorem.) One can use the maps $f_{k}$ and $g_{k}$ to construct the following two diagrams:

$$
\mathbb{Z} \longrightarrow \Omega \mathrm{DI}_{0} \stackrel{f_{0}}{\longrightarrow} \mathrm{DI}_{1} \stackrel{f_{1}}{\longrightarrow} \mathrm{B} \mathrm{DI}_{2} \stackrel{f_{2}}{\longrightarrow} \cdots \stackrel{f_{k-1}}{\longrightarrow} \mathrm{B}^{k-1} \mathrm{DI}_{k} \rightarrow \cdots
$$

in the homotopy case, and

$$
\mathbb{Z} \longrightarrow \mathbb{Z} \times B U \longrightarrow \Omega \mathrm{DU}_{0} \stackrel{g_{0}}{\longrightarrow} \mathrm{DU}_{1} \stackrel{g_{1}}{\longrightarrow} \mathrm{B} \mathrm{DU}_{2} \stackrel{g_{2}}{\longrightarrow} \cdots \stackrel{g_{k-1}}{\longrightarrow} \mathrm{B}^{k-1} \mathrm{DU}_{k} \rightarrow \cdots
$$

in the unitary case. The existence of these diagrams is explained in detail in [4, Section 12] - see especially Diagram 12.4. It is important to note that all the spaces in the above diagrams are infinite loop spaces, but the maps $f_{k}, g_{k}$ between them are not infinite loop maps. On the other hand, as is also discussed in detail in [4, Section 12], there exist infinite loop maps

$$
\alpha_{k}: \mathrm{B}^{k} \mathrm{DI}_{k+1} \longrightarrow \mathrm{B}^{k-1} \mathrm{DI}_{k} \quad \text { and } \quad \beta_{k}: \mathrm{B}^{k} \mathrm{DU}_{k+1} \longrightarrow \mathrm{B}^{k-1} \mathrm{DU}_{k}
$$

pointing in the opposite direction from $f_{k}$ and $g_{k}$. The maps $\alpha_{k}$ and $\beta_{k}$ derive from the symmetric power filtration and its $b u$-analogue (as per [4]). The point of Corollaries 6.2 and 7.2 is that they provide new, concrete evidence that there may be a direct relationship between these spectrum-level filtrations on the one hand, and the Goodwillie-Weiss towers on the other. To make the putative relationship more explicit, we state here the following conjecture, which is a variant of a conjecture made in [4].

Conjecture 1.2 The deloopings $f_{k}, g_{k}$ above can be chosen in such a way that the following maps are weak equivalences:

$$
f_{k} \circ \alpha_{k}+\alpha_{k+1} \circ f_{k+1} \quad \text { and } \quad g_{k} \circ \beta_{k}+\beta_{k+1} \circ g_{k+1}
$$

\footnotetext{
${ }^{2}$ In the case $k=0, \mathrm{~B}^{-1} X=\Omega X$.
} 
This conjecture is closely related to [4, Conjectures 12.1 and 12.5]. It says that there exist deloopings $\left(f_{k}, g_{k}\right)$ of the connecting maps in the Taylor towers that play a role of "contracting homotopies" for the "chain complexes" made of maps $\alpha_{k}$ and $\beta_{k}$. (See [4] for further explanation of this terminology.) Conjecture 1.2, if true, would imply that these chain complexes are exact. In the case of $\alpha_{k}$, the exactness is an old theorem of Kuhn [9] and Kuhn-Priddy [10], but even in this case it would be interesting to have a new proof, one that relates it to calculus. On the calculus side, the conjecture would imply that the homotopy spectral sequences associated with the Taylor tower of $S^{1}$ and of $B U(\mathbb{C})$ collapse at $E^{2}$. This is certainly believed to be true by experts, but a proof has so far been elusive.

The advance made in this paper is that we now know that the connecting maps in the Taylor tower are iterated loop maps of the required order. Hence the necessary deloopings exist.

Now we discuss the proofs of Theorems 6.1 and 7.1. The main ingredients are: Theorem 3.2, which presents a space of natural transformations as a homotopy fixed point spectrum; the Segal conjecture, which tells us that $\pi_{0}$ of a homotopy fixed point spectrum can sometimes be calculated; and the results of Arone-Dwyer [3] and Arone [2] on the derivatives of the identity functor and the BU(-) functor. Each of the cases presented in Theorems 6.1 and 7.1 has its own special features. Theorem 6.1 at the prime 2 is the simplest - it only requires a straightforward application of the Segal conjecture for finite groups, and we indicate its proof in Remark 6.8. The odd primary case of Theorem 6.1 is similar in principle, but is complicated to a surprising extent by the fact that we need to keep to odd-dimensional spheres. The action of the symmetric group on the derivatives is not obvious in this case, and we use some representation theory to analyze it. In Theorem 7.1, on the other hand, we have to come to terms with the homotopy fixed points of an action of a toral group, rather than just a finite group, and this complicates matters in a different way and necessitates the use of $p$-completion.

Section by section outline In Section 2 we recall the basic definitions of the model structure on categories of functors. We use the model structure to define the space hoNat $(-,-)$ of homotopy natural transformations and prove its basic properties. In Section 3, we calculate hoNat $(-,-)$ for inputs that are homogeneous functors, in order to prove Theorem 3.2. In Section 4, we give a first, easy delooping result by establishing Theorem 4.2. In Section 5, we use some representation theory to establish some technical results about the action of certain groups on certain complex Stiefel manifolds. These results will be needed in the both of the following sections, where we analyze spaces of homotopy natural transformations needed for Theorem 6.1 and Theorem 7.1 and prove the theorems. 
By a subgroup of a Lie group, we always mean a closed subgroup, except in the case of the group $I_{g}^{\prime}$ of Section 7.

\section{Preliminaries on model categories and spaces of natural transformations}

Throughout this paper, Top denotes the category of compactly generated spaces and $\mathrm{Top}_{*}$ the category of compactly generated pointed spaces. Both of these are closed symmetric monoidal model categories, with monoidal structure given by cartesian product (resp. smash product), weak equivalences given by weak homotopy equivalences, and fibrations by the Serre fibrations (see Hovey [8, 2.4.26]). The model structures are cofibrantly generated; in the case of Top, for instance, the generating cofibrations are the boundary inclusion maps

$$
S^{n-1} \hookrightarrow D^{n}
$$

and the generating acyclic cofibrations are the " $\times 0$ " endpoint inclusions

$$
D^{n} \hookrightarrow D^{n} \times I .
$$

For Top ${ }_{*}$, the generating (acyclic) cofibrations are the maps $U_{+} \rightarrow V_{+}$, where $U \rightarrow V$ is a generating (acyclic) cofibration for Top.

We will make heavy use of categories in which the morphism spaces are objects of Top or $\mathrm{Top}_{*}$.

Definition 2.1 A category enriched over Top is a category $\mathcal{C}$ provided with a topology on each one of the morphism sets $\operatorname{mor}_{\mathcal{C}}(i, j)$, in such a way that each morphism set is an object of Top and each composition map $\operatorname{mor}_{\mathcal{C}}(i, j) \times \operatorname{mor}_{\mathcal{C}}(j, k) \rightarrow \operatorname{mor}_{\mathcal{C}}(i, k)$ is continuous.

Remark 2.2 There is a similar definition of category enriched over Top ${ }_{*}$. In this case the morphism spaces have basepoints and the composition maps factor through continuous maps $\operatorname{mor}_{\mathcal{C}}(i, j) \wedge \operatorname{mor}_{\mathcal{C}}(j, k) \rightarrow \operatorname{mor}_{\mathcal{C}}(i, k)$. The forgetful functor $\operatorname{Top}_{*} \rightarrow$ Top allows any category enriched over Top ${ }_{*}$ to be treated as a category enriched over Top. Likewise, an enriched category can be treated as an ordinary (discrete) category by completely forgetting the topologies on the morphism sets.

Each of the categories Top and Top ${ }_{*}$ is enriched over itself. Similarly, let Spec be one of the standard topologically enriched closed symmetric monoidal model categories for spectra; for instance, Spec could be the category of topological symmetric spectra or topological orthogonal spectra (see Mandell, May, Schwede and Shipley [11]). Then 
Spec is enriched over Top ${ }_{*}$. The model structure of Spec is cofibrantly generated, with generating (acyclic) cofibrations given by the maps

$$
\Sigma_{k}^{\infty} K_{0} \hookrightarrow \Sigma_{k}^{\infty} K_{1}
$$

where $\Sigma_{k}^{\infty}$ is the $k$ th shift-suspension functor and $K_{0} \rightarrow K_{1}$ is a generating (acyclic) cofibration in $\mathrm{Top}_{*}$.

Clearly, if $\mathcal{C}$ is an enriched category, then $\mathcal{C}^{\text {op }}$ is an enriched category in a natural way. An enriched functor between two enriched categories is a functor between the underlying discrete categories that induces a continuous map on each morphism space (preserving basepoints when relevant). In general, we assume that functors are enriched whenever the categories involved are enriched.

For the rest of this section, $\mathcal{C}$ is a small category enriched over either Top or $\mathrm{Top}_{*}$, and $\mathcal{D}$ is Top, Top ${ }_{*}$, or Spec. We let $\mathcal{D}^{\mathcal{C}}$ denote the collection of (enriched) functors from $\mathcal{C}$ to $\mathcal{D}$. (Note that choosing $\mathcal{D}=$ Top only makes sense if $\mathcal{C}$ is enriched over Top.)

Definition 2.3 Let $F, G: \mathcal{C} \longrightarrow \mathcal{D}$ be two enriched functors. The space of natural transformations $\operatorname{Nat}_{\mathcal{C}}(F, G)$ is the usual set of natural transformations between the underlying discrete functors, topologized as the evident subspace of

$$
\prod_{i \in \operatorname{Ob}(\mathcal{C})} \operatorname{mor}_{\mathcal{D}}(F(i), G(i))
$$

The space of natural transformations is an example of an end. Similarly, one can define coends of enriched functors in the usual way. If $F$ and $G$ are enriched functors from $\mathcal{C}$ to $\mathcal{D}$, with $F$ contravariant and $G$ covariant, then the tensor product (coend) $F \otimes_{\mathcal{C}} G$ is defined to be the evident quotient of

$$
\bigoplus_{i \in \mathrm{Ob}(\mathcal{C})} F(i) \otimes G(i)
$$

Here, $\oplus$ (resp. $\otimes)$ stands for the direct sum (resp. monoidal product) in $\mathcal{D}$, so it is disjoint union (resp. Cartesian product) if $\mathcal{D}=$ Top and wedge sum (resp. smash product) if $\mathcal{D}$ is $\mathrm{Top}_{*}$ or $\mathrm{Spec}$. In constructing natural transformations and coends for enriched functors, the topologies on the morphism spaces of $\mathcal{C}$ do not play a role. If $\mathcal{C}$ is enriched over Top (as opposed to $\mathrm{Top}_{*}$ ) then there is a constant functor $1_{\mathcal{D}}: \mathcal{C} \longrightarrow \mathcal{D}$ sending every object of $\mathcal{C}$ to the $\otimes$-unit in $\mathcal{D}$ and every morphism to the identity map; this can be treated either as contravariant or covariant. In this case it is possible to consider $\operatorname{Nat}_{\mathcal{C}}\left(1_{\mathcal{D}}, G\right)$ or $1_{\mathcal{D}} \otimes_{\mathcal{C}} G$. If $\mathcal{D}$ is Top or Top ${ }_{*}$ the first construction gives the (inverse) limit of $G$; the second gives the colimit of $G$. Again, the morphism space topologies of $\mathcal{C}$ play no role in constructing the limit or colimit. 
Remark 2.4 The reader is invited to observe that if $\mathcal{C}$ is enriched over Top ${ }_{*}$ there is in general no interesting way to define $1_{\mathcal{D}}$ as a functor on $\mathcal{C}$ or to define the limit or colimit of a functor on $\mathcal{C}$. However, it is possible to construct a version of $1_{\mathcal{D}}$ if $\mathcal{C}$ is obtained from a category enriched over Top by adding disjoint basepoints to all of the morphism spaces and to define limits and colimits in this case. For this reason we will only consider limits and colimits of functors defined either on a category enriched over Top, or on a category enriched over Top $_{*}$ obtained from one enriched over Top by adding disjoint basepoints to all morphism spaces.

Using spaces of natural transformations as morphism spaces between the functors involved endows $\mathcal{D}^{\mathcal{C}}$ with the structure of an enriched category. In many situations this functor category also has the structure of a closed model category; we state this for the particular cases we need.

Proposition 2.5 Let $\mathcal{C}$ be a small category enriched over Top or $\mathrm{Top}_{*}$, and let $\mathcal{D}$ be either Top $_{*}$ or Spec. Then $\mathcal{D}^{\mathcal{C}}$ is a closed model category, where a morphism $F \longrightarrow G$ is a weak equivalence (resp. a fibration) if and only if the associated map $F(i) \rightarrow G(i)$ is a weak equivalence (resp. a fibration) in $\mathcal{D}$ for every object $i$ of $\mathcal{C}$.

Proof The case $\mathcal{D}=$ Spec is essentially the same as Schwede and Shipley [12, Theorem 7.2]. The case $\mathcal{D}=\mathrm{Top}_{*}$ is simpler and is proved similarly. One can also prove the proposition more directly, by verifying the conditions of Hovey [8, Theorem 2.1.19], which gives explicit conditions for a set of maps to generate a model structure.

Remark 2.6 The above model structure is cofibrantly generated, even finitely generated $[8,2.1 .3]$. In the case where $\mathcal{C}$ is enriched over Top and $\mathcal{D}$ is $\mathrm{Top}_{*}$ or Spec, the generating (acyclic) cofibrations are given by morphisms of the form

$$
K_{0} \wedge \operatorname{mor}_{\mathcal{C}}(i,-)_{+} \longrightarrow K_{1} \wedge \operatorname{mor}_{\mathcal{C}}(i,-)_{+}
$$

where $K_{0} \longrightarrow K_{1}$ is a generating (acyclic) cofibration in $\mathcal{D}$, and $i$ is an object of $\mathcal{C}$. (If $\mathcal{C}$ is enriched over Top $_{*}$, one omits the disjoint basepoints.) Note that if $K \in \mathcal{D}$ then, essentially by Yoneda's lemma, for any object $i \in \mathcal{C}$ and functor $G$ there is a natural homeomorphism

$$
\operatorname{Nat}\left(K \wedge \operatorname{mor}_{\mathcal{C}}(i,-)_{+}, G\right) \cong \operatorname{mor}_{\mathcal{D}}(K, G(i)) .
$$

If the morphism spaces in $\mathcal{C}$ are cofibrant, then the cofibrations in $\mathcal{D}^{\mathcal{C}}$ induce objectwise cofibrations of spaces or spectra. 
The natural transformation spaces and coend objects defined above are not adequate for our purposes, because they do not depend in a homotopy invariant way on the functors involved. We need to replace them with associated spaces of homotopy natural transformations or with homotopy coends. We do this by copying the usual technique for constructing derived functors $[8,1.3 .6]$. In general, when $X$ is an object of a model category, we use $c X$ (resp. $f X$ ) to denote a cofibrant (resp. fibrant) replacement for $X[8$, page 5].

Remark 2.7 All the model categories that we encounter are finitely generated [8, 2.1.17], and so the small object argument [8,2.1.14] guarantees that these replacements can be chosen to be functorial. The enhanced small object argument of Bousfield [6, $\S 5]$ and Farjoun [7, 1.B.1] guarantees that the replacements, when appropriate, can even be chosen to be functorial in an enriched sense.

Definition 2.8 Let $\mathcal{C}$ and $\mathcal{D}$ be as in Proposition 2.5, and let $F, G: \mathcal{C} \longrightarrow \mathcal{D}$ be functors. The space $\operatorname{hoNat}(F, G)$ of homotopy natural transformations from $F$ to $G$ is defined by

$$
\operatorname{hoNat}(F, G):=\operatorname{Nat}(c F, f G) .
$$

If $F$ is contravariant, the homotopy coend $F \otimes_{\mathcal{C}}^{\mathrm{h}} G$ is defined by

$$
F \otimes_{\mathcal{C}}^{\mathrm{h}} G:=c F \otimes_{\mathcal{C}} c G .
$$

Because of the functorial quality of cofibrant/fibrant replacement, the construction hoNat $(-,-)$ gives an enriched functor $\left(\mathcal{D}^{\mathcal{C}}\right)^{\mathrm{op}} \times \mathcal{D}^{\mathcal{C}} \rightarrow$ Top $_{*}$, and the construction $-\otimes_{\mathcal{C}}^{\mathrm{h}}$ - gives an enriched functor $\mathcal{D}^{\mathcal{C} \mathrm{op}} \times \mathcal{D}^{\mathcal{C}} \rightarrow \mathcal{D}$. The following lemma shows that these functors have the desired homotopy invariance property.

Lemma 2.9 Let $\mathcal{C}$ and $\mathcal{D}$ be as in Proposition 2.5, and let $F_{1}, F_{2}, G_{1}, G_{2}: \mathcal{C} \rightarrow \mathcal{D}$ be functors. If $\alpha: F_{2} \longrightarrow F_{1}$ and $\beta: G_{1} \longrightarrow G_{2}$ are weak equivalences of functors, then the induced map hoNat $\left(F_{1}, G_{1}\right) \longrightarrow \operatorname{hoNat}\left(F_{2}, G_{2}\right)$ is a weak equivalence of spaces. If $F_{1}$ and $F_{2}$ are contravariant, then the induced map $F_{2} \otimes_{\mathcal{C}}^{h} G_{1} \rightarrow F_{1} \otimes_{\mathcal{C}}^{h} G_{2}$ is a weak equivalence in $\mathcal{D}$.

Proof We only prove the result for homotopy natural transformations; the statement about homotopy coends is proved in a similar way. It is enough to prove that if in addition $F_{1}, F_{2}$ are cofibrant and $G_{1}, G_{2}$ are fibrant, then the induced map $\operatorname{Nat}\left(F_{1}, G_{1}\right) \longrightarrow$ $\operatorname{Nat}\left(F_{2}, G_{2}\right)$ is a weak equivalence of spaces. For this it is enough to show that if $G$ is fibrant, the functor $\operatorname{Nat}(-, G)$ takes weak equivalences between cofibrant functors into weak equivalences of spaces, and that if $F$ is cofibrant, the functor $\operatorname{Nat}(F,-)$ takes weak equivalences between fibrant functors into weak equivalences of spaces. 
We deal with the first statement in some detail because we will refer to this argument below. By Ken Brown's lemma $[8,1.1 .12]$ it is sufficient to prove that if $F^{\prime} \rightarrow F$ is an acyclic cofibration between cofibrant functors, then $\operatorname{Nat}(F, G) \rightarrow \operatorname{Nat}\left(F^{\prime}, G\right)$ is a weak equivalence. By a retract argument $[8,2.1 .18(\mathrm{e})]$, we can assume that $F^{\prime} \rightarrow F$ is a relative $J$-cell complex [8, 2.1.9], where $J$ is the set of generating acyclic cofibrations. Since the objects involved in these generating cofibrations have an appropriate finiteness property (see $[8,2.4 .2]$ and Remark 2.6 for $\mathcal{D}=\mathrm{Top}_{*}$ ), we can even assume that $F=\operatorname{colim}_{i} F_{i}$, where $F_{0}=F^{\prime}$ and $F_{i+1}$ is obtained from $F_{i}$ by a pushout diagram

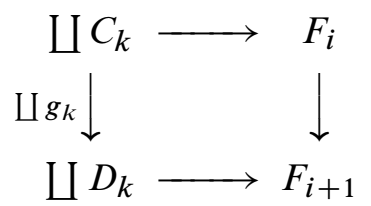

in which each map $g_{k}$ is a generating acyclic cofibration. Applying $\operatorname{Nat}(-, G)$ transforms such a pushout square of functors into a pullback square of spaces, and it is easy to check from the form of the generating acyclic cofibrations (Remark 2.6) that the map $\operatorname{Nat}\left(F_{i+1}, G\right) \rightarrow \operatorname{Nat}\left(F_{i}, G\right)$ is an acyclic fibration of spaces. Since $\operatorname{Nat}(F, G)=\lim _{i} \operatorname{Nat}\left(F_{i}, G\right)$, the desired result follows from the fact that the limit of a tower of fibrations in $\mathrm{Top}_{*}$ is equivalent to the homotopy limit.

The second statement above is proved similarly. The technique is to work inductively, constructing $F$ (up to retracts) from the initial object in terms of pushouts involving generating cofibrations $[8,2.1 .18(b)]$ and a concluding sequential colimit.

Almost all explicit calculations of spaces of natural transformations are based on the following lemma. Again, we are in the situation of Proposition 2.5.

Lemma 2.10 Suppose that $F$ is a functor of the form

$$
F(-)=K \wedge \operatorname{mor}_{\mathcal{C}}(i,-)_{+},
$$

where $K$ is a cofibrant object of $\mathcal{D}$ and $i$ is an object of $\mathcal{C}$. Suppose also that $G$ is a fibrant functor. Then

$$
\operatorname{hoNat}(F, G) \simeq \operatorname{Nat}(F, G) \cong \operatorname{mor}_{\mathcal{D}}(K, G(i)) .
$$

Proof It is a consequence of the definition of the model category structure that $F$ is a cofibrant functor. As in the proof of Lemma 2.9, this implies that the maps $\operatorname{Nat}(F, G) \rightarrow \operatorname{Nat}(c F, G)$ and $\operatorname{Nat}(c F, f G) \rightarrow \operatorname{Nat}(c F, G)$ are weak equivalences. The rest follows from the (enriched) Yoneda lemma (Remark 2.6). 
The above constructions can be used to obtain homotopy limits or homotopy colimits. Let $\mathcal{C}$ be a category enriched over Top, let $\mathcal{D}$ be either Top or Top ${ }_{*}$, and let $G: \mathcal{C} \longrightarrow \mathcal{D}$ be an enriched functor. Recall that $1_{\mathcal{D}}: \mathcal{C} \longrightarrow \mathcal{D}$ is the constant "unit" functor. We define the homotopy limit of $G$ by the formula

$$
\underset{\mathcal{C}}{\operatorname{holim}} G:=\operatorname{hoNat}_{\mathcal{C}}\left(1_{\mathcal{D}}, G\right)=\operatorname{Nat}\left(c 1_{\mathcal{D}}, f G\right) .
$$

Similarly, if $\mathcal{D}$ is Top, Top or Spec we define the homotopy colimit of $G$ by the formula

$$
\underset{\mathcal{C}}{\operatorname{hocolim}} G:=1_{\mathcal{D}} \otimes_{\mathcal{C}} c G=\operatorname{colim} c G
$$

Remark 2.11 The reader might wonder why we did not define the homotopy colimit of $G$ as $c 1_{\mathcal{D}} \otimes_{\mathcal{C}} c G$. It is not hard to check, using techniques from the proof of Lemma 2.9, that in our cases this alternative definition gives the same result up to weak equivalence as the definition above. Our definition, which was probably first used in a context like this by Farjoun, gives a notion that is slightly more convenient.

It is clear from Lemma 2.9 that homotopy limits and colimits respect weak equivalences. The following lemma gives a relationship between the two constructions.

Lemma 2.12 If $F$ is an object of $\mathcal{D}^{\mathcal{C}}$ with the property that each value $F(x)$ is a cofibrant object of $\mathcal{D}$, and if $Y$ is a fibrant object of $\mathcal{D}$, there is a natural weak equivalence of spaces

$$
\operatorname{mor}_{\mathcal{D}}(\operatorname{hocolim} F, Y) \simeq \operatorname{holim}_{x \in \mathcal{C}} \operatorname{mor}_{\mathcal{D}}(F(x), Y) .
$$

Proof This is obtained from the composite of the following chain of weak equivalences

$$
\begin{aligned}
\operatorname{mor}_{\mathcal{D}}(\operatorname{hocolim} F, Y) & \stackrel{\cong}{\rightarrow} \operatorname{mor}_{\mathcal{D}}(\operatorname{colim} c F, Y) \\
& \cong \lim _{x \in \mathcal{C}} \operatorname{mor}_{\mathcal{D}}(c F(x), Y) \\
& \cong \\
& \stackrel{\cong}{\rightarrow} \operatorname{holim}_{x \in \mathcal{C}} \operatorname{mor}_{\mathcal{D}}(c F(x), Y) \\
& \operatorname{holim}_{x \in \mathcal{C}} \operatorname{mor}_{\mathcal{D}}(F(x), Y) .
\end{aligned}
$$

Here, the first map is an isomorphism by the definition of homotopy colimit, the second map is an isomorphism by basic properties of limits and colimits, and the fourth map is a weak equivalence because the map $c F \longrightarrow F$ is a weak equivalence, and homotopy limits are homotopy invariant. It remains to show that the third map is a weak equivalence. This is proved by an inductive argument, as in the proof of Lemma 2.9; it 
relies on building $F$ from the initial object in terms of the generating cofibrations [8, 2.1.18(b)].

Next, we need to discuss the notion of a homotopy colimit of a diagram of functors; this is an instance of a homotopy left Kan extension, but we choose not to develop the general theory. Let $\mathcal{I}$ and $\mathcal{C}$ be small categories enriched over Top and Top ${ }_{*}$,

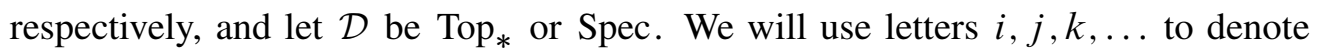
generic objects of $\mathcal{C}$ and $x, y, z$ to denote objects of $\mathcal{I}$. Suppose that we have a functor

$$
\mathcal{F}: \mathcal{I} \longrightarrow \mathcal{D}^{\mathcal{C}} .
$$

We think of $\mathcal{F}$ as a diagram of functors $\mathcal{C} \rightarrow \mathcal{D}$, indexed by $\mathcal{I}$. It is also possible to think of $\mathcal{F}$ as a diagram of functors $I \rightarrow \mathcal{D}$, indexed by $\mathcal{C}$; in fact, for each object $i$ of $\mathcal{C}$, there is a functor $\mathcal{F}_{i}: \mathcal{I} \rightarrow \mathcal{D}$ given by $\mathcal{F}_{i}(x)=(\mathcal{F}(x))(i)$.

Definition 2.13 Let $\mathcal{F}$ be an $\mathcal{I}$-diagram in $\mathcal{D}^{\mathcal{C}}$. The objectwise colimit (resp. objectwise homotopy colimit) of $\mathcal{F}$ is the object of $\mathcal{D}^{\mathcal{C}}$ which assigns to $i \in \mathcal{C}$ the object $\operatorname{colim} \mathcal{F}_{i}$ (resp. hocolim $\mathcal{F}_{i}$ ).

The (genuine) homotopy colimit of $\mathcal{F}$ is given by a slightly different construction. To begin with, let $\mathcal{C} \wedge \mathcal{I}_{+}$be the evident category whose set of objects is $\operatorname{Ob}(\mathcal{C}) \times \operatorname{Ob}(\mathcal{I})$ and where the space of morphisms from $(i, x)$ to $(j, y)$ is given by $\operatorname{mor}_{\mathcal{C}}(i, j) \wedge$ $\operatorname{mor}_{\mathcal{I}}(x, y)_{+}$. It is easy to see that there is an isomorphism of functor categories

$$
\left(\mathcal{D}^{\mathcal{C}}\right)^{\mathcal{I}} \cong \mathcal{D}^{\mathcal{C} \wedge \mathcal{I}_{+}}
$$

Using this isomorphism, we may endow $\left(\mathcal{D}^{\mathcal{C}}\right)^{\mathcal{I}}$ with a model structure in an obvious way (Proposition 2.5). This is a cofibrantly generated model structure, in which the generating (acyclic) cofibrations are morphisms of the form

$$
K_{0} \wedge \operatorname{mor}_{\mathcal{C}}(i,-) \wedge \operatorname{mor}_{\mathcal{I}}(x,-)_{+} \longrightarrow K_{1} \wedge \operatorname{mor}_{\mathcal{C}}(i,-) \wedge \operatorname{mor}_{\mathcal{I}}(x,-)_{+}
$$

where $i$ is an object of $\mathcal{C}, x$ is an object of $\mathcal{I}$, and $K_{0} \hookrightarrow K_{1}$ is a generating (acyclic) cofibration of $\mathcal{D}$.

Definition 2.14 Let $\mathcal{F}$ be an $\mathcal{I}$-diagram in $\mathcal{D}^{\mathcal{C}}$. The (genuine) homotopy colimit hocolim $\mathcal{F}$ of $\mathcal{F}$ is the objectwise colimit of $c \mathcal{F}$, where $c \mathcal{F}$ is the cofibrant replacement of $\mathcal{F}$ in the model structure on $\left(\mathcal{D}^{\mathcal{C}}\right)^{\mathcal{I}}$.

The following lemma is left as an exercise, along the lines of the proof of Lemma 2.12. 
Lemma 2.15 Let $\mathcal{F}$ be an $\mathcal{I}$-diagram in $\mathcal{D}^{\mathcal{C}}$ and $G$ an object of $\mathcal{D}^{\mathcal{C}}$. Then there is a natural weak equivalence of spaces

$$
\operatorname{hoNat}(\operatorname{hocolim} \mathcal{F}, G) \simeq \operatorname{holim}_{x \in \mathcal{I}} \operatorname{hoNat}(\mathcal{F}(x), G) .
$$

Our main point here is in the following lemma.

Lemma 2.16 If $\mathcal{F}$ is an $\mathcal{I}$-diagram in $\mathcal{D}^{\mathcal{C}}$, then the objectwise homotopy colimit of $\mathcal{F}$ is naturally weakly equivalent to the homotopy colimit of $\mathcal{F}$.

Proof Observe that if $h: \mathcal{G} \rightarrow \mathcal{G}^{\prime}$ is a weak equivalence between functors that are cofibrant in the model category structure on $\left(\mathcal{D}^{\mathcal{C}}\right)^{\mathcal{I}}$, then $h$ induces a weak equivalence between the objectwise colimits of these functors. This is proved by the technique in the proof of Lemma 2.9; it also follows from combining Lemma 2.15 with a Yoneda argument.

Let $c \mathcal{F}$ be a cofibrant replacement for $\mathcal{F}$ in the model structure on $\left(\mathcal{D}^{\mathcal{C}}\right)^{\mathcal{I}}$, and $c^{\prime} \mathcal{F}$ the functor obtained from $\mathcal{F}$ by, for each $i \in \mathcal{C}$, substituting for $\mathcal{F}_{i}$ its cofibrant replacement in the model structure on $\mathcal{D}^{\mathcal{I}}$. The natural maps $c \mathcal{F} \rightarrow \mathcal{F}$ and $c^{\prime} \mathcal{F} \rightarrow \mathcal{F}$ are both weak equivalences. Now consider the chain of weak equivalences

$$
c^{\prime} c c^{\prime} c \mathcal{F} \rightarrow c c^{\prime} c \mathcal{F} \rightarrow c^{\prime} c \mathcal{F} \rightarrow c \mathcal{F}
$$

and apply the objectwise colimit construction $\gamma$. As observed above, the composite of the rightmost two arrows becomes a weak equivalence, while from Lemma 2.9 it follows that the composite of the leftmost two arrows becomes a weak equivalence. As a consequence, all three arrows become weak equivalences. By definition, $\gamma(c \mathcal{F})$ is the homotopy colimit of $\mathcal{F}$. Again as above, the natural map $\gamma\left(c^{\prime} c \mathcal{F}\right) \rightarrow \gamma\left(c^{\prime} \mathcal{F}\right)$ is a weak equivalence, and the target is the objectwise homotopy colimit of $\mathcal{F}$.

Lemmas 2.15 and 2.16 have the following consequence, which we state explicitly.

Corollary 2.17 Let $\mathcal{I}$ and $\mathcal{C}$ be categories enriched over Top and $\mathrm{Top}_{*}$, respectively, and let $\mathcal{D}$ be $\mathrm{Top}_{*}$ or Spec. Let $\mathcal{F}$ be an $\mathcal{I}$-diagram in $\mathcal{D}^{\mathcal{C}}$ and $F$ the objectwise homotopy colimit of $\mathcal{F}$. Then for any object $G$ of $\mathcal{D}^{\mathcal{C}}$ there is a natural weak equivalence

$$
\operatorname{hoNat}(F, G) \simeq \operatorname{holim}_{x \in \mathcal{I}} \operatorname{hoNat}(\mathcal{F}(x), G) .
$$

We next list various adjointness properties of the hoNat construction. These are proved by routine arguments using the techniques employed above. In the first statement $\Sigma^{\infty}$ is the suspension functor from pointed spaces to spectra, and $\Omega^{\infty}$ its (derived) adjoint, the zero'th space functor. 
Lemma 2.18 Let $F: \mathcal{C} \rightarrow \mathrm{Top}_{*}$ and $G: \mathcal{C} \rightarrow$ Spec be enriched functors. Then there is a natural weak equivalence hoNat ${ }_{\mathcal{C}}\left(\Sigma^{\infty} F, G\right) \simeq \operatorname{hoNat}_{\mathcal{C}}\left(F, \Omega^{\infty} G\right)$.

If $A$ and $B$ are spectra, let mapspec $(A, B)$ denote the (derived) mapping spectrum of maps from $A$ to $B$. The smash products in the following lemmas are derived smash products.

Lemma 2.19 Let $F, G: \mathcal{C} \rightarrow$ Spec be enriched functors, and $A$ a spectrum. Then there is a natural weak equivalence

$$
\operatorname{hoNat}_{\mathcal{C}}(A \wedge F, G) \simeq \operatorname{hoNat}_{\mathcal{C}}(F, \operatorname{mapspec}(A, G)) .
$$

Lemma 2.20 Let $F, G: \mathcal{C} \rightarrow$ Top $_{*}$ be enriched functors, and $A$ a space. Then there is a natural weak equivalence $\operatorname{hoNat}_{\mathcal{C}}(A \wedge F, G) \simeq \operatorname{hoNat}_{\mathcal{C}}(F, \operatorname{mor}(A, G))$.

Finally, we need to recall the definition and basic properties of (homotopy) right Kan extensions; again, we content ourselves with treating a special case. Let $\mathcal{C}$ be a category enriched over Top, and let $\mathcal{C}_{0} \subset \mathcal{C}$ be a subcategory. For simplicity, we will assume that $\mathcal{C}_{0}$ and $\mathcal{C}$ have the same sets of objects, but this is not an essential assumption. In this case, $\mathcal{D}$ is Top $_{*}$.

Definition 2.21 Suppose that $\mathcal{D}=\mathrm{Top}_{*}$, and let $G: \mathcal{C}_{0} \rightarrow \mathcal{D}$ be an enriched functor. The homotopy right Kan extension of $G$ from $\mathcal{C}_{0}$ to $\mathcal{C}$, denoted hoInd $\mathcal{C}_{\mathcal{C}_{0}}^{\mathcal{C}} G$, is defined by the following formula

$$
\operatorname{hoInd}_{\mathcal{C}_{0}}^{\mathcal{C}} G(i):= \begin{cases}\operatorname{hoNat}_{j \in \mathcal{C}_{0}}\left(\operatorname{mor}_{\mathcal{C}}(i, j)_{+}, G(j)\right) & \mathcal{D}=\text { Top }_{*} \\ \operatorname{hoNat}_{j \in \mathcal{C}_{0}}\left(\Sigma^{\infty} \operatorname{mor}_{\mathcal{C}}(i, j)_{+}, G(j)\right) & \mathcal{D}=\text { Spec }\end{cases}
$$

The fact that hoInd $\mathcal{C}_{\mathcal{C}_{0}}^{\mathcal{C}} G$ is a functor on $\mathcal{C}$ depends on the fact that functors on $\mathcal{C}_{0}$ such as $\operatorname{mor}_{\mathcal{C}}(i,-)_{+}$can be supplied with natural cofibrant replacements, as mentioned in Remark 2.7. The main point that we need to make about the functor hoInd $\mathcal{C}_{0}^{\mathcal{C}}: \mathcal{D}^{\mathcal{C}_{0}} \longrightarrow$ $\mathcal{D}^{\mathcal{C}}$ is that it is a homotopy adjoint to the restriction functor $\operatorname{Res}_{\mathcal{C}_{0}}^{\mathcal{C}}: \mathcal{D}^{\mathcal{C}} \longrightarrow \mathcal{D}^{\mathcal{C}_{0}}$. What this means concretely is stated in the following lemma.

Lemma 2.22 Suppose that $\mathcal{D}=$ Top $_{*}$, and let $F: \mathcal{C} \longrightarrow \mathcal{D}$ and $G: \mathcal{C}_{0} \longrightarrow \mathcal{D}$ be functors. There is a natural weak equivalence

$$
\operatorname{hoNat}_{\mathcal{C}}\left(F, \operatorname{hoInd}_{\mathcal{C}_{0}}^{\mathcal{C}} G\right) \simeq \operatorname{hoNat}_{\mathcal{C}_{0}}\left(\operatorname{Res}_{\mathcal{C}_{0}}^{\mathcal{C}} F, G\right)
$$


Proof Homotopy right Kan extension preserves fibrant functors, so it is enough to show that if $F$ is cofibrant and $G$ is fibrant then the natural map

$$
\operatorname{Nat}_{\mathcal{C}}\left(F, \operatorname{hoInd}_{\mathcal{C}_{0}}^{\mathcal{C}} G\right) \longrightarrow \text { hoNat }_{\mathcal{C}_{0}}\left(\operatorname{Res}_{\mathcal{C}_{0}}^{\mathcal{C}} F, G\right)
$$

is a weak equivalence. As in the proof of Lemma 2.9, it is enough to show that the map is an equivalence when $F$ is the free functor

$$
F(-)=K \wedge \operatorname{mor}_{\mathcal{C}}(i,-)_{+}
$$

determined by an object $i \in \mathcal{C}$ and a cofibrant object $K$ of $\mathcal{D}$. In this case, it follows from the Yoneda lemma and Lemma 2.20 that the domain of $(2-1)$ is $\operatorname{Map}_{*}\left(K, \operatorname{hoInd}_{\mathcal{C}_{0}}^{\mathcal{C}} G(i)\right)$, while the target is

$$
\operatorname{hoNat}_{j \in \mathcal{C}_{0}}\left(K \wedge \operatorname{mor}_{\mathcal{C}}(i, j)_{+}, G(j)\right),
$$

which by Lemma 2.20 and the definition of homotopy right Kan extension is equivalent to $\operatorname{Map}_{*}\left(K\right.$, hoInd $\left._{\mathcal{C}_{0}}^{\mathcal{C}} G(i)\right)$. By inspection the map in question is in fact an equivalence. This completes the proof.

\section{Natural transformations of homogeneous functors}

In this section, we calculate the space of (homotopy) natural transformations between homogeneous functors in orthogonal calculus. Since our main application requires working over complex vector spaces, this section is written in the complex setting. However, all the work in this section can be done equally well over the reals.

Let $\mathcal{J}$ be the category of finite-dimensional complex vector spaces with inner product and linear isometric inclusions. The category $\mathcal{J}$ is enriched over Top in a natural way, with the morphism spaces $\operatorname{mor}_{\mathcal{J}}(V, W)$ being the appropriate complex Stiefel manifolds. Following Weiss [13, Section 1] we define a sequence of categories $\mathcal{J}_{n}$, enriched over $\operatorname{Top}_{*}$. All of the categories $\mathcal{J}_{n}$ have the same set of objects as $\mathcal{J}$. To define the morphism space $\operatorname{mor}_{\mathcal{J}_{n}}(V, W)$ in $\mathcal{J}_{n}$, consider the vector bundle $\gamma(V, W)$ over $\operatorname{mor}_{\mathcal{J}}(V, W)$ with the total space consisting of pairs $(f, x)$ where $f \in \operatorname{mor}_{\mathcal{J}}(V, W)$ and $x \in \operatorname{coker}(f)$. Let $\gamma_{n}(V, W)$ be the vector bundle obtained from $\gamma(V, W)$ by tensoring each fiber with $\mathbb{C}^{n}$. The morphism space $\operatorname{mor}_{\mathcal{J}_{n}}(V, W)$ from $V$ to $W$ in $\mathcal{J}_{n}$ is defined to be the Thom space of $\gamma_{n}(V, W)$.

There are inclusions of categories

$$
\mathcal{J}_{0} \subseteq \mathcal{J}_{1} \subseteq \mathcal{J}_{2} \subseteq \cdots
$$

Notice that $\mathcal{J}_{0}$ is the category obtained from $\mathcal{J}$ by adding a disjoint basepoint to each morphism complex. In other words, $\operatorname{mor}_{\mathcal{J}_{0}}(V, W) \cong \operatorname{mor}_{\mathcal{J}}(V, W)_{+}$. Let $\mathcal{D}$ be $\operatorname{Top}_{*}$, 
or Spec. There is an isomorphism of categories of enriched functors $\mathcal{D}^{\mathcal{J}} \cong \mathcal{D}^{\mathcal{J}_{0}}$, and we will switch between these two categories without further comment.

Lemma 3.1 Let $F: \mathcal{J} \longrightarrow$ Spec be a continuous functor. There is a natural equivalence

$$
\operatorname{hoNat}_{\mathcal{J}}\left(\Sigma^{\infty} S^{n V}, F(V)\right) \simeq\left(\text { hoInd }_{\mathcal{J}_{0}}^{\mathcal{J}_{n}} \Omega^{\infty} F\right)\left(\mathbb{C}^{0}\right)
$$

Proof The result is implicit in the reasoning of Weiss [13, Sections 1 and 2], although it is not made explicit there. The idea of the proof is to note that although $S^{n V}$ is not a representable functor when considered as a functor on $\mathcal{J}$ or $\mathcal{J}_{0}$, it is the restriction of a representable functor on $\mathcal{J}_{n}$. Indeed,

$$
S^{n V} \cong \operatorname{mor}_{\mathcal{J}_{n}}\left(\mathbb{C}^{0}, V\right)
$$

We obtain a sequence of natural weak equivalences,

$$
\begin{aligned}
\operatorname{hoNat}_{\mathcal{J}_{0}}\left(\Sigma^{\infty} S^{n V}, F(V)\right) & \simeq \operatorname{hoNat}_{\mathcal{J}_{0}}\left(S^{n V}, \Omega^{\infty} F(V)\right) \\
& \simeq \operatorname{hoNat}_{\mathcal{J}_{0}}\left(\operatorname{Res}_{\mathcal{J}_{0}}^{\mathcal{J}_{n}} \operatorname{mor}_{\mathcal{J}_{n}}\left(\mathbb{C}^{0}, V\right), \Omega^{\infty} F(V)\right) \\
& \simeq \operatorname{hoNat}_{\mathcal{J}_{n}}\left(\operatorname{mor}_{\mathcal{J}_{n}}\left(\mathbb{C}^{0}, V\right), \operatorname{hoInd}_{\mathcal{J}_{0}}^{\mathcal{J}_{n}} \Omega^{\infty} F(V)\right) \\
& \simeq\left(\operatorname{hoInd}_{\mathcal{J}_{0}}^{\mathcal{J}_{n}} \Omega^{\infty} F\right)\left(\mathbb{C}^{0}\right) .
\end{aligned}
$$

Here the second equivalence follows from Lemma 2.18, the third equivalence follows from Lemma 2.22, and the last equivalence is just the Yoneda lemma (Lemma 2.10).

Now we are ready to state and prove the general result about spaces of natural transformations between homogeneous functors. Let $U(n)$ denote the linear, inner-product preserving automorphisms of $\mathbb{C}^{n}$. If $C$ is a spectrum with an action of $U(n)$, we let $M_{C, n}$ denote the functor $\mathcal{J} \rightarrow$ Spec which sends a vector space $V$ to $\left(C \wedge \Sigma^{\infty} S^{n V}\right)_{\tilde{h} U(n)}$. This is a homogeneous functor in the unitary calculus.

Theorem 3.2 Let $C$ and $D$ be spectra with actions of $U(n)$ and $U(k)$, respectively. Then

$$
\operatorname{hoNat}_{\mathcal{J}}\left(M_{C, n}, M_{D, k}\right) \simeq \operatorname{Map}\left(C,\left(D \wedge \operatorname{mor}_{\mathcal{J}}\left(\mathbb{C}^{n}, \mathbb{C}^{k}\right)_{+}\right)_{\tilde{h} U(k)}\right)^{\tilde{h} U(n)}
$$

Proof Using Corollary 2.17 and Lemma 2.19, we can write

$$
\begin{aligned}
\operatorname{hoNat}_{\mathcal{J}}\left(M_{C, n}, M_{D, k}\right) & \simeq \operatorname{hoNat}_{\mathcal{J}}\left[C \wedge \Sigma^{\infty} S^{n V}, M_{D, k}(V)\right]^{\tilde{h} U(n)} \\
& \simeq \operatorname{hoNat}_{\mathcal{J}}\left[\Sigma^{\infty} S^{n V}, \operatorname{mapspec}\left(C, M_{D, k}(V)\right)\right]^{\tilde{h} U(n)}
\end{aligned}
$$

Algebraic $\&$ Geometric Topology, Volume 8 (2008) 
We next calculate the final term without the superscript $\tilde{h} U(n)$. By Lemma 3.1, this is equivalent to calculating the value at $V=\mathbb{C}^{0}$ of the functor

$$
\operatorname{hoInd}_{\mathcal{J}_{0}}^{\mathcal{J}_{n}} \Omega^{\infty} \operatorname{mapspec}\left(C, M_{D, k}(V)\right) \text {. }
$$

The key to this is contained in [13, Example 5.7]. (See also [13, Notation 2.5] for the correspondence.) This reference contains the calculation

$$
\operatorname{hoInd}_{\mathcal{J}_{0}}^{\mathcal{J}_{n}} \Omega^{\infty} M_{D, k}(V) \simeq \begin{cases}* & n>k \\ \Omega^{\infty}\left(D \wedge S^{k V}\right)_{\tilde{h} U(k-n)} & 0 \leq n \leq k\end{cases}
$$

where as usual everything is expressed as a functor of the vector-space variable $V$, and $U(k-n)$ fixes the first $n$ coordinates of $\mathbb{C}^{k}$. Let $F(C, D)$ be the value of (3-1) at $V=\mathbb{C}^{0}$; we wish to produce a weak equivalence

$$
F(C, D) \simeq \operatorname{Map}\left(C, D_{\widetilde{h} U(k-n)}\right) \simeq \Omega^{\infty} \operatorname{mapspec}\left(C, D_{\widetilde{h} U(k-n)}\right) .
$$

It is easy to produce a map $\lambda$ between the two objects involved, and to observe, by substituting $V=\mathbb{C}^{0}$ in (3-2), that $\lambda$ is a weak equivalence for $C=S^{0}$. Noting that $F(C, D) \simeq F\left(S^{0}, C^{\#} \wedge D\right)$ allows the observation to be extended to the case in which $C$ is a finite spectrum. Now the constructions in (3-3) convert homotopy colimits in $C$ to homotopy limits, so the class of spectra for which $\lambda$ is a weak equivalence is closed under homotopy colimits; since the class includes all finite spectra, it must include all spectra.

The proof of the theorem is completed by observing that $\operatorname{mor}_{\mathcal{J}}\left(\mathbb{C}^{n}, \mathbb{C}^{k}\right) \cong U(k) / U(k-$ $n$ ) and applying Shapiro's lemma.

The following is a consequence of Theorem 3.2 and dualization.

Corollary 3.3 If $C$ is a finite spectrum induced up from a $K$-spectrum $B$ for some $K \subseteq U(n)$, that is, $C \simeq U(n)_{+} \wedge_{K} B$, then

$$
\operatorname{hoNat}_{\mathcal{J}}\left(M_{C, n}, M_{D, k}\right) \simeq\left(B^{\#} \wedge\left(\Sigma^{\infty} \operatorname{mor}_{\mathcal{J}}\left(\mathbb{C}^{n}, \mathbb{C}^{k}\right)_{+} \wedge D\right)_{\tilde{h} U(k)}\right)^{\tilde{h} K}
$$

where the smash product has the diagonal action of $K$ via the actions of $K$ on $B^{\#}$ and on $\operatorname{mor}_{\mathcal{J}}\left(\mathbb{C}^{n}, \mathbb{C}^{k}\right)$.

Remark 3.4 Theorem 3.2 is analogous to a well-known formula (due to Goodwillie) in the homotopy calculus of functors from spaces to spectra. Let $C$ and $D$ be spectra with actions of $\Sigma_{n}$ and $\Sigma_{k}$, respectively, and let $F, G$ : Top ${ }_{*} \rightarrow$ Spec be the functors 
that assign to $X$ the spectra $\left(C \wedge X^{\wedge n}\right)_{\tilde{h} \Sigma_{n}}$ and $\left(D \wedge X^{\wedge k}\right)_{\tilde{h} \Sigma_{k}}$. Then $\operatorname{hoNat}(F, G)$ is the space

$$
\Omega^{\infty} \operatorname{mapspec}\left(C,\left(D \wedge \operatorname{Surj}(\mathbf{k}, \mathbf{n})_{+}\right)_{\tilde{h} \Sigma_{k}}\right)^{\tilde{h} \Sigma_{n}}
$$

Here $\operatorname{Surj}(\mathbf{k}, \mathbf{n})$ is the set of surjections from a set with $k$ elements to a set with $n$ elements. As in the statement of Theorem 3.2, "mapspec" is the mapping spectrum in the enriched category Spec.

The proof is somewhat simpler than the proof of Theorem 3.2. Indeed, standard manipulations with adjunctions together with a smallness argument show that

$$
\operatorname{hoNat}\left(\left(C \wedge X^{\wedge n}\right)_{\tilde{h} \Sigma_{n}},\left(D \wedge X^{\wedge k}\right)_{\tilde{h} \Sigma_{k}}\right)
$$

is equivalent to

$$
\Omega^{\infty} \operatorname{mapspec}\left(C,\left(D \wedge \operatorname{hoNatspec}\left(\Sigma^{\infty} X^{\wedge n}, \Sigma^{\infty} X^{\wedge k}\right)\right)_{\tilde{h} \Sigma_{k}}\right)^{\tilde{h} \Sigma_{n}} .
$$

where "hoNatspec" denotes an appropriately defined spectrum of natural transformations between spectrum-valued functors. To show that (3-5) is equivalent to (3-4) it is only necessary to observe that hoNatspec $\left(\Sigma^{\infty} X^{\wedge n}, \Sigma^{\infty} X^{\wedge k}\right)$ is equivalent to $\Sigma^{\infty} \operatorname{Surj}(\mathbf{k}, \mathbf{n})_{+}$, that is, every natural stable map between iterated smash powers of $X$ is given by (sums of) iterated diagonals. This completes the proof.

Remark 3.5 Let $I_{p}$ be the fibre of the rationalization map from the $p$-local stable sphere to the rational sphere; $I_{p}$ is a Moore spectrum of type $\left(\mathbb{Z} / p^{\infty},-1\right)$. Bousfield localization on the category of spectra with respect to the mod $p$ stable homotopy functor is given by $L_{p}(X)=\operatorname{mapspec}\left(I_{p}, X\right)$. This functor is idempotent (because $I_{p} \wedge I_{p} \simeq I_{p}$ ) and agrees with Bousfield localization with respect to mod $p$ homology on spectra that are bounded below. The functor $L_{p}$ is what is ordinarily called the stable $p$-completion functor.

For the rest of this paragraph, write $\hat{X}=L_{p}(X)$ for the $p$-completion of $X$. In the situation of Theorem 3.2, write $E=\left(D \wedge \operatorname{mor}_{\mathcal{J}}\left(\mathbb{C}^{n}, \mathbb{C}^{k}\right)_{+}\right)_{\tilde{h} U(k)}$, so that Theorem 3.2 reads

$$
\operatorname{hoNat}_{\mathcal{J}}\left(M_{C, n}, M_{D, k}\right) \simeq \operatorname{Map}(C, E)^{\tilde{h} U(n)} .
$$

We would like to prove that

$$
\operatorname{hoNat}_{\mathcal{J}}\left(\widehat{M}_{C, n}, \widehat{M}_{D, k}\right) \simeq \operatorname{Map}(\widehat{C}, \widehat{E})^{\tilde{h} U(n)}
$$


Observe first of all that $\widehat{M}_{D, k}=\operatorname{mapspec}\left(I_{p}, M_{D, k}\right)$, so by Lemma 2.19 and inspection we get

$$
\begin{aligned}
\operatorname{hoNat}_{\mathcal{J}}\left(M_{C, n}, \hat{M}_{D, k}\right) & \simeq \operatorname{hoNat}_{\mathcal{J}}\left(I_{p} \wedge M_{C, n}, M_{D, k}\right) \\
& \simeq \operatorname{hoNat}_{\mathcal{J}}\left(M_{I_{p} \wedge C, n}, M_{D, k}\right) \\
& \simeq \operatorname{Map}\left(I_{p} \wedge C, E\right)^{\tilde{h} U(n)} \\
& \simeq \operatorname{Map}(C, \hat{E})^{\tilde{h} U(n)} .
\end{aligned}
$$

It remains to show that the $p$-completion map $M_{C, n} \rightarrow \widehat{M}_{C, n}$ induces an equivalence on hoNat $\mathcal{J}\left(-, \widehat{M}_{D, k}\right)$, and that the $p$-completion map $C \rightarrow \widehat{C}$ induces an equivalence on $\operatorname{Map}(-, \hat{E})$. The proofs are similar; we will do the first. If $F$ is the fibre of $M_{C, n} \rightarrow \hat{M}_{C, n}$, it is enough to prove that hoNat $\mathcal{J}\left(F, \hat{M}_{D, k}\right)$ is contractible. But there are weak equivalences

$$
\begin{aligned}
\operatorname{hoNat}_{\mathcal{J}}\left(F, \hat{M}_{D, k}\right) & \simeq \operatorname{hoNat}_{\mathcal{J}}\left(F, \operatorname{mapspec}\left(I_{p}, \hat{M}_{D, k}\right)\right) \\
& \simeq \operatorname{hoNat}_{J}\left(I_{p} \wedge F, \widehat{M}_{D, k}\right),
\end{aligned}
$$

and $I_{p} \wedge F$ is trivial, since $I_{p}$ is $p$-torsion and $F=\operatorname{mapspec}\left(S_{\mathbb{Z}[1 / p]}^{0}, M_{C, n}\right)$ is $\mathbb{Z}[1 / p]-$ local.

\section{Infinite delooping of functors into spaces}

In this section, we prove that certain functors from spaces to spaces in fact take their values in infinite loop spaces.

As a preliminary, we prove the following.

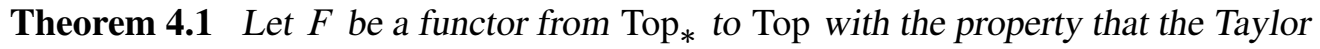
tower of $F$ consists of two layers, $\left(D_{n} F\right)(X)$ and $\left(D_{k} F\right)(X)$, where $n<k<2 n$. Then $F$ takes values in infinite loop spaces.

Proof Suppose that we have $\left(D_{n} F\right)(X)=\Omega^{\infty}\left(C \wedge X^{\wedge n}\right)_{\tilde{h} \Sigma_{n}}$ and $\left(D_{k} F\right)(X)=$ $\Omega^{\infty}\left(D \wedge X^{\wedge k}\right) \tilde{h}_{\Sigma_{k}}$. There is a natural fibration

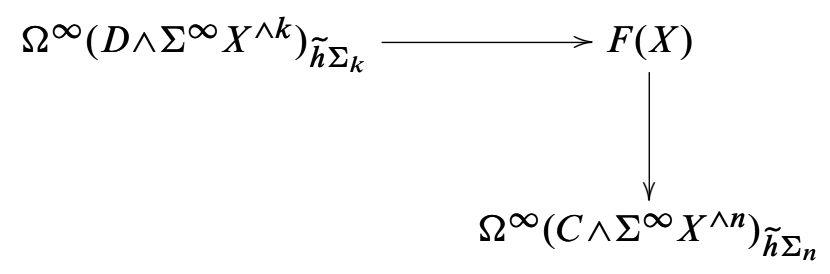

Algebraic $8 \mathcal{G}$ Geometric Topology, Volume 8 (2008) 
and the fibration is induced by a natural transformation

$$
\gamma(X): \Omega^{\infty}\left(C \wedge \Sigma^{\infty} X^{\wedge n}\right)_{\tilde{h} \Sigma_{n}} \rightarrow \Omega^{\infty}\left(\Sigma D \wedge \Sigma^{\infty} X^{\wedge k}\right)_{\tilde{h} \Sigma_{k}},
$$

or alternatively, taking the adjoint,

$$
\tilde{\gamma}(X): \Sigma^{\infty} \Omega^{\infty}\left(C \wedge \Sigma^{\infty} X^{\wedge n}\right)_{\tilde{h} \Sigma_{n}} \rightarrow \Sigma\left(D \wedge \Sigma^{\infty} X^{\wedge k}\right)_{\tilde{h} \Sigma_{k}} .
$$

The layers of the Taylor tower of the functor $\Sigma^{\infty} \Omega^{\infty}\left(C \wedge \Sigma^{\infty} X^{\wedge n}\right) \tilde{h}_{n}$ have the form $\left.\left[\left(C \wedge \Sigma^{\infty} X^{\wedge n}\right)\right)_{\tilde{h} \Sigma_{n}}\right] \tilde{h} \Sigma_{i}$. If $i>1$, this functor has degree greater than $k$, and there are no nontrivial natural transformations to $\left(\Sigma D \wedge \Sigma^{\infty} X^{\wedge k}\right)_{\tilde{h} \Sigma_{k}}$ by Remark 3.4. Thus $\tilde{\gamma}$ factors through the projection to the lowest homogeneous layer of $\Sigma^{\infty} \Omega^{\infty}\left(C \wedge \Sigma_{n}\right.$ $\left.\Sigma^{\infty} X^{\wedge n}\right)$, that is, through the evaluation map:

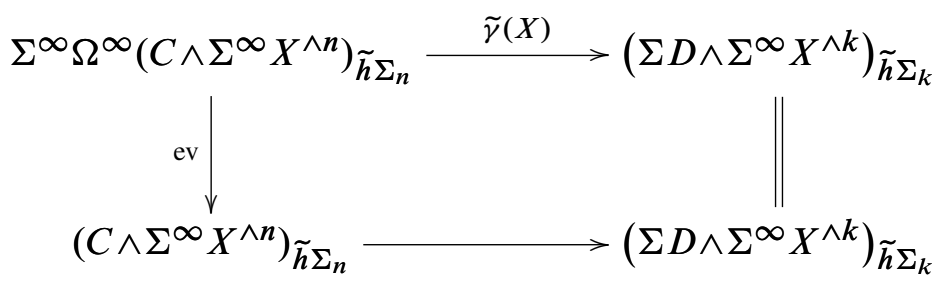

This says precisely that $\gamma(X)$ is an infinite loop map, and thus $F$ takes values in infinite loop spaces.

By similar arguments, one obtains the following generalization.

Theorem 4.2 Let $F$ be a functor from Top $_{*}$ to Top with the property that the Taylor tower of $F$ consists of layers $\left(D_{i} F\right)(X)$ for $n \leq i<k<2 n$. Let $G$ be a functor defined by taking the fiber of a natural transformation $\gamma: F \rightarrow \Omega^{\infty}\left(C_{k} \wedge \Sigma_{k} X^{\wedge k}\right)$. Then $G$ takes values in infinite loop spaces.

\section{Representation theory}

Let $p$ be prime. In later sections, we will be studying natural transformations of homogeneous spectrum-valued functors on finite-dimensional complex vector spaces. In particular, we will need to look at natural transformations from functors of degree $i p^{k}(1<i \leq p)$ to those of degree $p^{k+1}$, and in Section 3, we found that such natural transformations involve the space mor $\mathcal{J}\left(\mathbb{C}^{i p^{k}}, \mathbb{C}^{p^{k+1}}\right)$ of linear isometric inclusions $\mathbb{C}^{i p^{k}} \rightarrow \mathbb{C}^{p^{k+1}}$, along with its action of $U\left(i p^{k}\right)$ (from the right) and $U\left(p^{k+1}\right)$ (from the left). Our applications actually involve coefficient spectra with actions that are induced up to an action of a unitary group from smaller, sometimes finite groups, and 
we will then need to understand the actions of these smaller isotropy groups on spheres formed as the one-point compactifications of complex vector spaces. This section establishes the necessary prerequisites.

Note that $\Sigma_{p^{k}} \subseteq U\left(p^{k}\right)$ as permutations of the standard basis vectors. Let $\Delta_{k} \subseteq \Sigma_{p^{k}}$ be the usual transitive elementary abelian $p$-subgroup, and let $\Gamma_{k} \subseteq U\left(p^{k}\right)$ be the usual projective elementary abelian $p$-subgroup, an extension

$$
1 \rightarrow S^{1} \rightarrow \Gamma_{k} \rightarrow(\mathbb{Z} / p)^{2 k} \rightarrow 1 .
$$

(See, for example, Arone and Lesh [4, Section 10].) There is a diagram of inclusions

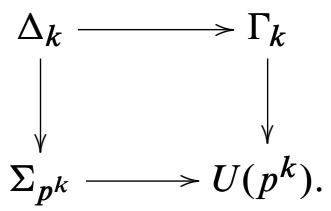

We write $\operatorname{mor}_{\mathcal{J}}\left(\mathbb{C}^{i p^{k}}, \mathbb{C}^{p^{k+1}}\right)$ as the right coset space $H_{i} \backslash U\left(p^{k+1}\right)$, where $H_{i}=$ $U\left(p^{k+1}-i p^{k}\right) \subseteq U\left(p^{k+1}\right)$ is the subgroup of $U\left(p^{k+1}\right)$ that fixes the first $i p^{k}$ standard basis vectors. We regard $\Sigma_{i} \prec \Gamma_{k}$ as a subgroup of $U\left(p^{k+1}\right)$ in the obvious way, as acting on the subspace $\mathbb{C}^{i p^{k}} \subseteq \mathbb{C}^{p^{k+1}}$ spanned by the first $i p^{k}$ basis vectors. In later sections, we need to understand $H_{i} \backslash U\left(p^{k+1}\right)$ as a space with a left action of $\Sigma_{i}\left\langle\Gamma_{k} \times \Gamma_{k+1}\right.$ by $(\phi, \gamma) \cdot H_{i} g=H_{i} \phi g \gamma^{-1}$. (Note that $\Sigma_{i} \imath \Gamma_{k} \subseteq U\left(p^{k+1}\right)$ commutes with $H_{i}$.) Suppose that $I_{g} \subseteq \Sigma_{i} \prec \Gamma_{k} \times \Gamma_{k+1}$ is the isotropy group of $H_{i} g$.

Lemma 5.1 If $i>1$, then the compositions

$$
\begin{aligned}
& I_{g} \hookrightarrow \Sigma_{i} \prec \Gamma_{k} \times \Gamma_{k+1} \rightarrow \Gamma_{k+1} \\
& I_{g} \hookrightarrow \Sigma_{i} \prec \Gamma_{k} \times \Gamma_{k+1} \rightarrow \Sigma_{i} \prec \Gamma_{k}
\end{aligned}
$$

are both monomorphisms, where the second map in each composition is projection to a factor.

Proof The element $(\phi, \gamma) \in \Sigma_{i} \prec \Gamma_{k} \times \Gamma_{k+1}$ is in $I_{g}$ exactly when there exists $h \in H_{i}$ such the $g \gamma g^{-1}=\phi h$. If $(\phi, \gamma)$ is in the kernel of $(5-1)$, then $\gamma=e$, so $\phi h=e$, which implies that (5-1) is a monomorphism because the intersection of the subgroups $\Sigma_{i} \prec \Gamma_{k}$ and $H_{i}$ of $U\left(p^{k+1}\right)$ is trivial.

Suppose that $(\phi, \gamma) \in I_{g}$ is a nonidentity element. We know that $\gamma \neq e$ from the previous paragraph. We wish to show that $\phi \neq e$, and because $\phi=e$ would imply the existence of $h \in H_{i}$ such that $g \gamma g^{-1}=h$, it is sufficient to show that no nonidentity element $\gamma \in \Gamma_{k+1}$ can be conjugate to an element $h \in H_{i}$. We do this by comparing 
the eigenspaces of $h$ and $\gamma$, with the starting point that if $h \in H_{i}$ is not the identity, then the eigenspace of $h$ associated to the eigenvalue 1 has dimension at least $i p^{k}$ (which is strictly greater than $p^{k}$ ) but less than $p^{k+1}$. We will show that, by contrast, elements of $\Gamma_{k+1}$ either have $p$ eigenspaces, each of dimension exactly $p^{k}$, or else they have a single eigenspace, of dimension $p^{k+1}$. This will finish the proof of the lemma.

To study the eigenvalues and eigenspaces of a nonidentity element $\gamma \in \Gamma_{k+1}$, we first consider a special case. Let $\delta$ be the element of $\Gamma_{k+1}$ corresponding to the product of $p$-cycles

$$
(1, \ldots, p)(p+1, \ldots, 2 p) \ldots\left((p-1) p^{k}+1, \ldots, p^{k+1}\right) .
$$

Let $\zeta$ be a primitive $p$ th root of unity. Direct calculation establishes that the eigenvalues of $\delta$ are $1, \zeta, \zeta^{2}, \ldots, \zeta^{p-1}$, each with multiplicity $p^{k}$, so the eigenspaces of $\delta$ are of dimension exactly $p^{k}$.

Recall that $\Gamma_{k+1}$ can be written as an extension

$$
1 \rightarrow S^{1} \rightarrow \Gamma_{k+1} \rightarrow(\mathbb{Z} / p)^{2 k+2} \rightarrow 1 .
$$

Then all elements in the component $\delta S^{1}$ of $\Gamma_{k+1}$ have eigenspaces of dimension exactly $p^{k}$, because multiplication by an element $z \in S^{1}$ just multiplies the eigenvalues by $z$. To finish, we observe that the normalizer of $\Gamma_{k+1}$ in $U\left(p^{k+1}\right)$, namely $S p_{2 k+2}\left(\mathbb{F}_{p}\right)$, acts transitively on the set of nonidentity components of $\Gamma_{k+1}$, and so all of the elements of all of the nonidentity components have eigenspaces of dimension exactly $p^{k}$. On the other hand, elements of the identity component all have a single eigenspace, of dimension $p^{k+1}$. This finishes the proof.

The action of $\Sigma_{i} \succ \Gamma_{k} \times \Gamma_{k+1}$ on $H_{i} \backslash U\left(p^{k+1}\right)$ can be restricted to an action of $\Sigma_{i} \prec \Delta_{k} \times \Delta_{k+1}$. Let $\widetilde{I}_{g} \subseteq \Sigma_{i} \curlywedge \Delta_{k} \times \Delta_{k+1}$ be the isotropy group of $H_{i} g$ under this restricted action, that is, $\left.\widetilde{I}_{g}=I_{g} \cap\left(\Sigma_{i}\right\rangle \Delta_{k} \times \Delta_{k+1}\right)$. From Lemma 5.1, we know that the composite

$$
\tilde{I}_{g} \hookrightarrow \Sigma_{i} \prec \Delta_{k} \times \Delta_{k+1} \rightarrow \Sigma_{i} \prec \Delta_{k}
$$

is a monomorphism; let $J_{g} \subseteq \Sigma_{i} \prec \Delta_{k}$ be its image. In Section 6, we need to know about the representation of $J_{g}$ that is obtained by restriction of the wreath product representation of $\Sigma_{i} \Delta_{k}$ in $U\left(i p^{k}\right)$. Because the result we need is representationtheoretic, we make the representations explicit, rather than implicit as in the earlier part of this section.

Proposition 5.2 Let $\sigma: \Sigma_{i} \curlywedge \Delta_{k} \hookrightarrow U\left(i p^{k}\right)$ be the embedding by the wreath product representation. Let $J_{g} \subseteq \Sigma_{i}$ 々 $\Delta_{k}$ be as above. Then the restriction of $\sigma$ to $J_{g}$ is a sum of copies of the regular representation of $J_{g}$. 
Proof Let $\tau: \Delta_{k+1} \rightarrow U\left(p^{k+1}\right)$ be the regular representation, and regard $U\left(i p^{k}\right)$ as the subgroup of $U\left(p^{k+1}\right)$ that acts on the span of the first $i p^{k}$ basis vectors, while fixing the orthogonal complement. If $(\phi, \delta) \in \widetilde{I}_{g}$, then there exists $h \in H_{i}$ with

$$
\sigma(\phi) h=g \tau(\delta) g^{-1}
$$

Note that $\phi$ is the image of $(\phi, \delta)$ under the isomorphism from $\tilde{I}_{g}$ to $J_{g}$. We want to prove that the trace of $\sigma(\phi)$ is 0 if $\phi \neq e$, which is sufficient to characterize the restriction of $\sigma$ to $J_{g}$ as being a multiple of the regular representation of $J_{g}$.

Since $\sigma(\phi)$ is a permutation matrix, it is sufficient to prove that $\phi$ acts on $\left\{1, \ldots, i p^{k}\right\}$ without fixed points. Suppose that $\phi=\left(s ; \phi_{1} \ldots, \phi_{i}\right)$ where $s \in \Sigma_{i}$ and $\phi_{1}, \ldots, \phi_{i} \in$ $\Delta_{k}$. If $s \neq e \in \Sigma_{i}$, then we must have $i=p$ and $s$ is a $p$-cycle, because $\phi$ has order $p$. Thus $\phi$ has no fixed points on $\left\{1, \ldots, i p^{k}\right\}$.

On the other hand, suppose that $s=e \in \Sigma_{i}$, so that $\phi=\left(e ; \phi_{1} \ldots, \phi_{i}\right)$, and suppose that $\phi$ is not the identity. It is sufficient to establish that no $\phi_{j}$ is the identity of $\Delta_{k}$, because every nonidentity element of $\Delta_{k}$ acts on $p^{k}$ objects without fixed points, establishing that $\phi$ acts on $\left\{1, \ldots, i p^{k}\right\}$ without fixed points. Our strategy is to consider the two sides of (5-3) and to show that if any $\phi_{j}$ is the identity of $\Delta_{k}$, then the dimensions of the pointwise-fixed subspaces of $\sigma(\phi) h$ and $g \tau(\delta) g^{-1}$ acting on $\mathbb{C}^{p^{k+1}}$ do not match.

On the right-hand side of (5-3), we know that $\tau(\delta)$ is acting on $\mathbb{C}^{p^{k+1}}$ through the regular representation of $\Delta_{k+1}$. Further, because $\phi$ is not the identity, $\delta$ cannot be the identity either. Thus $\tau(\delta)$ has a pointwise-fixed subspace of dimension exactly $\left[\Delta_{k+1}:\langle\delta\rangle\right]=p^{k}$.

On the left-hand side of (5-3), $\sigma(\phi)$ and $h$ act on orthogonal complements in $\mathbb{C}^{p^{k+1}}$. Thus the element $\sigma(\phi) h \in U\left(p^{k+1}\right)$ has a pointwise-fixed subspace of dimension at least as large that of $\sigma(\phi)$ acting on $\mathbb{C}^{i p^{k}} \cong \mathbb{C}^{p^{k}} \oplus \cdots \oplus \mathbb{C}^{p^{k}}$. Since $\phi=\left(e ; \phi_{1} \ldots, \phi_{i}\right)$, the dimension of the pointwise-fixed subspace of $\sigma(\phi)$ is just the sum of the dimensions of the pointwise-fixed subspaces of the individual elements $\phi_{j}$ acting on their respective summands $\mathbb{C}^{p^{k}}$. But $\phi_{j}$ acts through the regular representation of $\Delta_{k}$, so nonidentity elements $\phi_{j}$ have a pointwise fixed subspace of dimension $\left[\Delta_{k}:\langle\delta\rangle\right]=p^{k-1}$. Thus $\sigma(\phi) h$ has a pointwise fixed subspace of $\mathbb{C}^{p^{k+1}}$ of dimension at least $i p^{k-1}$. If one of the $\phi_{j}$ is actually the identity of $\Delta_{k}$, then in fact $\sigma(\phi) h$ has a pointwise-fixed subspace of $\mathbb{C}^{p^{k+1}}$ of dimension at least $p^{k}+(i-1) p^{k-1}$. Since $i>1$, this is greater than the dimension of the pointwise-fixed subspace of $\tau(\delta)$, a contradiction. 


\section{Delooping structure maps: the homotopy case}

Let $p$ be an odd prime. In this section, we study the connecting maps between the layers of the Goodwillie tower of the $p$-localization of the identity functor on Top ${ }_{*}$. It is proved in Arone-Mahowald [5] that when evaluated at odd spheres, the layers of this tower are trivial except at powers of $p$. This gives structure maps in the tower that connect the $p^{k}$ th layer directly to the $p^{k+1}$ st layer. Let $\mathrm{DI}_{k}(X)$ be the $p^{k}$ th layer of the $p$-localized Taylor tower for the identity functor evaluated at $X$. In Arone-Lesh [4, Conjecture 12.1] it is suggested that the structure map $\operatorname{DI}_{k}\left(S^{1}\right) \rightarrow B \mathrm{DI}_{k+1}\left(S^{1}\right)$ deloops $k$ times. In this section, we prove this conjecture, though we do not establish the specific delooping that was suggested in [4]. The strategy is to show that the natural transformation giving the structure map for odd spheres is a retract by natural infinite loop maps from a natural transformation that deloops $k$ times for very general reasons. There are analogous results for $p=2$, and in this case there is no restriction that the dimension of the sphere be odd. Most of the section deals with the odd-primary case. The case $p=2$ is much easier, and we discuss it in Remark 6.8.

As explained in the introduction, an obvious difficulty with our strategy arises from the fact that it is only when evaluated at appropriate spheres that the structure map for the Taylor tower of the identity connects the $p^{k}$ th layer directly to the $p^{k+1}$ st layer. As a consequence, we cannot study this map by using results about natural transformations of homogeneous functors of spaces. We need a way to study the aspects of this Taylor tower that are natural only in odd spheres. Our approach is to precompose the identity functor on $\mathrm{Top}_{*}$ with the functor $\mathcal{X}: V \mapsto S^{1} \wedge S^{V}$ from finite-dimensional complex vector spaces to pointed spaces. When we evaluate the Taylor tower for the identity at the odd-dimensional sphere $S^{1} \wedge S^{V}$, the resulting tower is natural in $V$ and consists of layers that are homogeneous functors of $V$ in the sense of Weiss's orthogonal calculus. General calculus reasoning establishes that the Weiss tower of $\mathcal{X}$, evaluated at $V$, is the same as the Goodwillie tower of the identity, evaluated at $\mathcal{X}(V)$. Further, when $p$-localized, the tower has the $p^{k}$ th layer connected directly to the $p^{k+1}$ st layer.

Theorem 6.1 Let $\mathcal{X}$ be the functor from finite-dimensional vector spaces over $\mathbb{C}$ (over $\mathbb{R}$ if $p=2$ ) given by $\mathcal{X}(V)=\left(S^{1} \wedge S^{V}\right)_{(p)}\left(\right.$ resp. $\mathcal{X}(V)=\left(S^{V}\right)_{(2)}$ if $\left.p=2\right)$. Then the $k$-fold looping map

$$
\Omega^{k}: \operatorname{hoNat}\left(\mathrm{B}^{k} \mathrm{D}_{p^{k}} \mathcal{X}, \mathrm{B}^{k+1} \mathrm{D}_{p^{k+1}} \mathcal{X}\right) \rightarrow \operatorname{hoNat}\left(\mathrm{D}_{p^{k}} \mathcal{X}, \mathrm{B} \mathrm{D}_{p^{k+1}} \mathcal{X}\right)
$$

is surjective on $\pi_{0}$.

Our main interest lies in the following corollary. 
Corollary 6.2 Let $p$ be a prime and let $n=p^{k}$. Let $X$ be an odd-dimensional sphere (or any sphere, if $p=2$ ). Let $\operatorname{DI}_{k}(X)$ be the $p^{k}$ th layer of the $p$-localized Goodwillie tower of the identity evaluated at $X$. Then the connecting map

$$
\kappa_{k}: \mathrm{DI}_{k}(X) \rightarrow \mathrm{BDI}_{k+1}(X)
$$

admits a $k$-fold delooping in the homotopy category of spaces.

The special case $X=S^{1}$ of the corollary was conjectured in [4].

Fix an odd prime $p$, and assume throughout that all spaces are localized at $p$. The proof of Theorem 6.1 follows immediately from the following three statements. (The case $p=2$ is handled in Remark 6.8.)

Theorem 6.3 Let $\Delta_{k}$ be the transitive elementary abelian $p$-subgroup of $\Sigma_{p^{k}} o b$ tained by letting $\left(\mathbf{F}_{p}\right)^{k}$ act on itself by translation. For any nonnegative integer $d$, the $d$-fold looping map

$$
\begin{gathered}
\operatorname{hoNat}\left[Q\left(\left(S^{1 \wedge} S^{V}\right)_{\tilde{h} \Delta_{k}}^{\wedge p^{k}}\right), \quad Q\left(\left(S^{1} \wedge S^{V}\right)_{\tilde{h} \Delta_{k+1}}^{\wedge p^{k+1}}\right)\right] \\
\operatorname{hoNat}\left[\Omega^{d} Q\left(\left(S^{1} \wedge S^{V}\right)_{\tilde{h} \Delta_{k}}^{\wedge p^{k}}\right), \quad \Omega^{d} Q\left(\left(S^{1} \wedge S^{V}\right)_{\tilde{h} \Delta_{k+1}^{\wedge}}^{\wedge p^{k+1}}\right)\right]
\end{gathered}
$$

is an epimorphism on $\pi_{0}$.

Lemma 6.4 For each $k$, the functor $\mathrm{D}_{p^{k}} \mathcal{X}(V)$ is a retract of the functor

$$
\Omega^{k} Q\left(\left(S^{1} \wedge S^{V}\right) \underset{\widetilde{h} \Delta_{k}}{\wedge p^{k}}\right)
$$

Moreover, both the inclusion and the retraction are infinite loop maps.

Lemma 6.5 Suppose that $\mathcal{C}$ is a small category, that $\tilde{\mathcal{Y}}_{1}$ and $\tilde{\mathcal{Y}}_{2}$ are functors from $\mathcal{C}$ to Top $_{*}$, and that for $i=1,2$, there are natural transformations $\iota_{i}: \mathcal{Y}_{i} \rightarrow \tilde{\mathcal{Y}}_{i}$ and $\rho_{i}: \tilde{\mathcal{Y}}_{i} \rightarrow \mathcal{Y}_{i}$ such that $\rho_{i} l_{i}$ is a weak equivalence on $\mathcal{Y}_{i}(C)$ for each object $C$ in $\mathcal{C}$. Let $d$ be a positive integer, and suppose that the $d$-fold looping map

$$
\operatorname{hoNat}\left(\tilde{\mathcal{Y}}_{1}, \tilde{\mathcal{Y}}_{2}\right) \longrightarrow \operatorname{hoNat}\left(\Omega^{d} \tilde{\mathcal{Y}}_{1}, \Omega^{d} \tilde{\mathcal{Y}}_{2}\right)
$$

is an epimorphism on $\pi_{0}$. Then the same is true for the $d$-fold looping map.

$$
\operatorname{hoNat}\left(\mathcal{Y}_{1}, \mathcal{Y}_{2}\right) \longrightarrow \operatorname{hoNat}\left(\Omega^{d} \mathcal{Y}_{1}, \Omega^{d} \mathcal{Y}_{2}\right) \text {. }
$$

We will give the proofs of these three statements in reverse order. 
Proof of Lemma 6.5 Consider the maps

$$
\operatorname{hoNat}\left(\mathcal{Y}_{1}, \mathcal{Y}_{2}\right) \longrightarrow \operatorname{hoNat}\left(\tilde{\mathcal{Y}}_{1}, \tilde{\mathcal{Y}}_{2}\right) \stackrel{p}{\longrightarrow} \operatorname{hoNat}\left(\mathcal{Y}_{1}, \mathcal{Y}_{2}\right)
$$

where the first map is induced by the maps $\rho_{1}$ and $\iota_{2}$, while $p$ is induced by $\rho_{2}$ and $\iota_{1}$. Because $\rho_{i} l_{i}$ is a weak equivalence, the composed map above is a weak equivalence. In particular, $p$ is surjective on $\pi_{0}$. Similarly, there are maps

$$
\operatorname{hoNat}\left(\Omega^{d} \mathcal{Y}_{1}, \Omega^{d} \mathcal{Y}_{2}\right) \longrightarrow \operatorname{hoNat}\left(\Omega^{d} \tilde{\mathcal{Y}}_{1}, \Omega^{d} \tilde{\mathcal{Y}}_{2}\right) \stackrel{p_{d}}{\longrightarrow} \operatorname{hoNat}\left(\Omega^{d} \mathcal{Y}_{1}, \Omega^{d} \mathcal{Y}_{2}\right)
$$

and $p_{d}$ is surjective on $\pi_{0}$. There is a commutative square

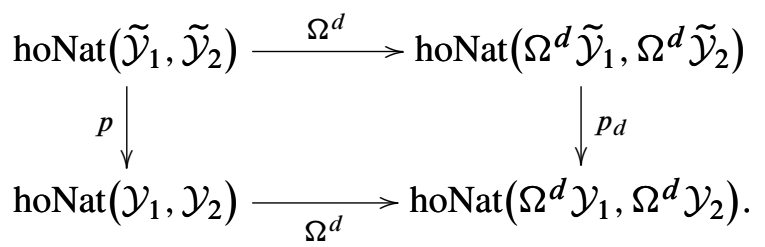

In this diagram, the vertical maps are surjective on $\pi_{0}$ by the above discussion, while the top horizontal map is surjective on $\pi_{0}$ by hypothesis. It follows that the bottom horizontal map is surjective on $\pi_{0}$.

Proof of Lemma 6.4 We need to recall from Arone and Dwyer [3] the description of the spectrum associated to the infinite loop space $\mathrm{DI}_{k}\left(S^{1} \wedge S^{V}\right)=\mathrm{D}_{p^{k}} \mathcal{X}(V)$. Notice that for any pointed space $Y$, the group $\mathrm{GL}_{k}\left(\mathbf{F}_{p}\right)$ acts on $\Sigma^{\infty}\left(Y^{\wedge p^{k}}\right)_{\tilde{h} \Delta_{k}}$ because $\mathrm{GL}_{k}\left(\mathbf{F}_{p}\right)$ is the Weyl group of $\Delta_{k}$ in $\Sigma_{p^{k}}$. Let $\epsilon_{k} \in \mathrm{GL}_{k}\left(\mathbf{F}_{p}\right)$ be the Steinberg idempotent. It is proved in [3] that if $Y$ is an odd sphere, then

$$
\begin{aligned}
\operatorname{DI}_{k}(Y) & \simeq \Omega^{\infty}\left[S^{-k} \wedge \epsilon_{k} \cdot \Sigma^{\infty}\left(Y^{\wedge p^{k}}\right)_{\tilde{h} \Delta_{k}}\right] \\
& \simeq \Omega^{k} \Omega^{\infty}\left[\epsilon_{k} \cdot \Sigma^{\infty}\left(Y^{\wedge p^{k}}\right)_{\tilde{h} \Delta_{k}}\right]
\end{aligned}
$$

where $\epsilon_{k} \cdot \Sigma^{\infty}\left(Y^{\wedge} p^{k}\right)_{\tilde{h} \Delta_{k}}$ denotes the summand of $\Sigma^{\infty}\left(Y^{\wedge} p^{k}\right)_{\tilde{h} \Delta_{k}}$ given by the mapping telescope of $\epsilon_{k}$. Note that the retraction of $\Sigma^{\infty}\left(Y^{\wedge p^{k}}\right)_{\tilde{h} \Delta_{k}}$ onto the summand $\epsilon_{k} \cdot \Sigma^{\infty}\left(Y^{\wedge p^{k}}\right)_{\tilde{h} \Delta_{k}}$ has nothing to do with $Y$ itself, but only with the action of $\mathrm{GL}_{k}\left(\mathbf{F}_{p}\right)$ on $\Sigma^{\infty}\left(Y^{\wedge p^{k}}\right)_{\tilde{h} \Delta_{k}}$, which is natural in $Y$. Thus when we take $Y=\mathcal{X}(V)$, there is a natural retraction

$$
\rho(V): \Sigma^{\infty}\left(\mathcal{X}(V)^{\wedge p^{k}}\right)_{\widetilde{h} \Delta_{k}} \longrightarrow \epsilon_{k} \cdot \Sigma^{\infty}\left(\mathcal{X}(V)^{\wedge p^{k}}\right)_{\widetilde{h} \Delta_{k}}
$$

and the corresponding inclusion,

$$
\iota(V): \epsilon_{k} \cdot \Sigma^{\infty}\left(\mathcal{X}(V)^{\wedge p^{k}}\right)_{\tilde{h} \Delta_{k}} \longrightarrow \Sigma^{\infty}\left(\mathcal{X}(V)^{\wedge p^{k}}\right)_{\tilde{h} \Delta_{k}}
$$


is also natural in $V$. Since $\rho(V)$ and $\iota(V)$ are maps of spectra, they induce infinite loop maps on the associated infinite loop spaces.

The rest of the section is devoted to the proof of Theorem 6.3. We will need the following standard manipulations for the proof.

Lemma 6.6 Let $H \subseteq G$ where $G$ is finite, let $X$ be an $H$-spectrum, and let $Y$ be a $G$-spectrum. Then the following are equivalences of $G$-spectra.

(1) $Y \wedge\left(G_{+} \wedge_{H} X\right) \simeq G_{+} \wedge_{H}(Y \wedge X)$, where the left has the diagonal action of $G$ and the right has the left action of $G$.

(2) $G_{+} \wedge_{H} X \simeq \operatorname{Map}_{H}(G, X)$.

To set up the proof of Theorem 6.3, consider $\mathcal{X}(V)^{\wedge p^{k}}=\left(S^{1} \wedge S^{V}\right)^{\wedge p^{k}}$ as a $\Sigma_{p^{k}}-$ space. We write it as $S^{p^{k}} \wedge S^{p^{k} V}$, where $\Sigma_{p^{k}}$ acts on $S^{p^{k}}$ by permuting the factors of $S^{p^{k}} \simeq\left(S^{1}\right)^{\wedge p^{k}}$ and acts on $S^{p^{k} V}=S^{\mathbb{C}^{p^{k}} \otimes V}$ by permuting the basis vectors of $\mathbb{C}^{p^{k}}$. To position ourselves to apply Theorem 3.2, we rearrange the target of (6-1) using adjointness to the form

(6-2) hoNat $\left[S^{d} \wedge \Sigma^{\infty} \Omega^{\infty}\left(S^{-d} \wedge \Sigma^{\infty} S^{p^{k}} \wedge S^{p^{k} V}\right)_{\tilde{h} \Delta_{k}}, \quad \Sigma^{\infty}\left(S^{1} \wedge S^{V}\right)_{\tilde{h} \Delta_{k+1}}^{\wedge p^{k+1}}\right]$

and we manipulate the homogeneous layers of both the source and the target of (6-2) to make the coefficient spectra apparent. This is easy for the target, which actually is a homogeneous functor, of degree $p^{k+1}$. To find the coefficient spectrum, we need to be looking at homotopy orbits of an action of $U\left(p^{k+1}\right)$, so we induce up from $\Delta_{k+1}$ to $U\left(p^{k+1}\right)$ and find that the target of (6-2) is represented by the coefficient spectrum $U\left(p^{k+1}\right)_{+} \wedge_{\Delta_{k+1}} \Sigma^{\infty} S^{p^{k+1}}$.

However, the source of (6-2) is not a homogeneous functor, so we look at its homogeneous layers and calculate their coefficient spectra.

Lemma 6.7 The functor

$$
S^{d} \wedge \Sigma^{\infty} \Omega^{\infty}\left(S^{-d} \wedge \Sigma^{\infty} S^{p^{k}} \wedge S^{p^{k} V}\right)_{\tilde{h} \Delta_{k}}
$$

has nontrivial homogeneous layers only in dimensions that are multiples of $p^{k}$. The $i p^{k}$ th layer is represented by the spectrum

$$
U\left(i p^{k}\right)+\wedge \Sigma_{i} \Delta_{k}\left[S^{-(i-1) d} \wedge \Sigma^{\infty} S^{i p^{k}}\right]
$$

where $\Delta_{k}$ acts trivially on $S^{-(i-1) d}$ and $\Sigma_{i}$ acts on $S^{-(i-1) d}$ by the dual of the one-point compactification of $d$ copies of the reduced standard representation. 
Proof It is well known (see, for example, Ahearn and Kuhn [1]) that the $i$ th homogeneous layer of the functor from spectra to spectra $X \mapsto \Sigma^{\infty} \Omega^{\infty} X$ is $\left(\Sigma^{\infty} X^{\wedge i}\right)_{\tilde{h} \Sigma_{i}}$. It follows that the $i p^{k}$ th homogeneous layer of (6-3) is

$$
S^{d} \wedge\left[\left(S^{-d} \wedge \Sigma^{\infty} S^{p^{k}} \wedge S^{p^{k} V}\right)_{\tilde{h} \Delta_{k}}\right]_{\tilde{h} \Sigma_{i}}^{\wedge i} \simeq S^{d} \wedge\left(S^{-i d} \wedge \Sigma^{\infty} S^{i p^{k}} \wedge S^{i p^{k} V}\right)_{\tilde{h} \Sigma_{i} \Delta_{k}}
$$

and that the other layers are trivial. Here $\left.\Sigma_{i}\right\rangle \Delta_{k}$ acts on $S^{-i d}$ by the dual of the one-point compactification of the action of $\Sigma_{i} \imath \Delta_{k}$ on $\mathbb{R}^{i} \otimes \mathbb{R}^{d}$ that projects $\Sigma_{i}$ 々 $\Delta_{k}$ to $\Sigma_{i}$ and then acts on $\mathbb{R}^{i}$ by the standard representation. The standard action of $\Sigma_{i}$ on $\mathbb{R}^{i}$ splits off a one-dimensional trivial representation, and so $S^{i d}$ is $\Sigma_{i}$-equivariantly equivalent to $S^{d} \wedge S^{(i-1) d}$, where $\Sigma_{i}$ acts trivially on $S^{d}$ and acts on $S^{(i-1) d}$ by the one-point compactification of $d$ copies of the reduced standard representation. Thus the $i$ th homogeneous layer of the source of (6-2) is equivalent to

$$
\left(S^{-(i-1) d} \wedge \Sigma^{\infty} S^{i p^{k}} \wedge S^{i p^{k} V}\right)_{\tilde{h} \Sigma_{i} \Delta_{k}}
$$

Finally, as before, we induce up to $U\left(i p^{k}\right)$ to find the coefficient spectra, and we find that the $i p^{k}$ th layer of (6-3) is represented by the spectrum

$$
U\left(i p^{k}\right)+\wedge \Sigma_{i} \Delta_{k}\left[S^{-(i-1) d} \wedge \Sigma^{\infty} S^{i p^{k}}\right]
$$

Proof of Theorem 6.3 for odd primes We consider (6-2), and we let $Z(V)$ denote the cofiber of the map

$$
\begin{gathered}
S^{d} \wedge \Sigma^{\infty} \Omega^{\infty}\left(S^{-d} \wedge \Sigma^{\infty} S^{p^{k}} \wedge S^{p^{k} V}\right)_{\tilde{h} \Delta_{k}} \\
\downarrow \\
S^{0} \wedge \Sigma^{\infty} \Omega^{\infty}\left(S^{0} \wedge \Sigma^{\infty} S^{p^{k}} \wedge S^{p^{k} V}\right)_{\tilde{h} \Delta_{k}}
\end{gathered}
$$

that induces the adjoint form of (6-1). Noting that the source of (6-1) has the form of the target evaluated for $d=0$, we see that we need to show that

$$
\operatorname{hoNat}\left(\Sigma^{-1} Z(V), \Sigma^{\infty}\left(S^{1} \wedge S^{V}\right)_{\tilde{h} \Delta_{k+1}}^{\wedge p^{k+1}}\right)
$$

is $0-$ connected.

We use the homogeneous layers of $Z(V)$ for the calculation. We define $\operatorname{Cof}_{i, d}=$ $\operatorname{Cof}\left(S^{0} \rightarrow S^{(i-1) d}\right)$, where $S^{0}$ is included as the north and south poles, and we let $\operatorname{Cof}_{i, d}^{\#}$ denote its Spanier-Whitehead dual. By applying Lemma 6.7 twice (once with $d=0$ ) and taking the appropriate cofiber, we find that the $i p^{k}$ th homogeneous layer 
of $Z(V)$ is represented by the spectrum

$$
U\left(i p^{k}\right)_{+} \wedge_{\Sigma_{i} \imath \Delta_{k}}\left[\Sigma \operatorname{Cof}_{i, d}^{\#} \wedge \Sigma^{\infty} S^{i p^{k}}\right]
$$

as $i$ runs through positive integers. (All other layers are contractible.)

Let $F_{i}$ be the spectrum given by

$$
F_{i}:=\left[\left(\Sigma^{-1} \operatorname{Cof}_{i, d} \wedge\left(S^{-1}\right)^{\wedge i p^{k}}\right) \wedge\left(\operatorname{mor}_{\mathcal{J}}\left(\mathbb{C}^{i p^{k}}, \mathbb{C}^{p^{k+1}}\right)_{+} \wedge \Sigma^{\infty} S^{p^{k+1}}\right)_{\tilde{h} \Delta_{k+1}}\right]^{\tilde{h} \Sigma_{i} 2 \Delta_{k}}
$$

Recalling that the target of (6-2) is a homogeneous functor, and using Corollary 3.3, we find that hoNat $\left(\Sigma^{-1} Z(V), \Sigma^{\infty}\left(S^{1} \wedge S^{V}\right)_{\tilde{h} \Delta}^{\wedge p^{k+1}}\right)$ can be built up by principal fibrations with fibers of the form $\Omega^{\infty} \Sigma F_{i}$ where $i \geq 1$. In fact, mor $\mathcal{J}\left(\mathbb{C}^{i p^{k}}, \mathbb{C}^{p^{k+1}}\right)_{+}$ is contractible if $i>p$, and $\operatorname{Cof}_{i, d}$ is contractible if $i=1$, so it suffices to show that $F_{i}$ is $(-1)$-connected for $1<i \leq p$.

Our analysis of $F_{i}$ depends on understanding the interaction of the left action of $\Delta_{k+1}$ and the right action of $\Sigma_{i} \curlywedge \Delta_{k}$ on $\operatorname{mor}_{\mathcal{J}}\left(\mathbb{C}^{i p^{k}}, \mathbb{C}^{p^{k+1}}\right)$. We use the notation of Section 5, writing $\operatorname{mor}_{\mathcal{J}}\left(\mathbb{C}^{i p^{k}}, \mathbb{C}^{p^{k+1}}\right)$ as the right coset space $H_{i} \backslash U\left(p^{k+1}\right)$, where $H_{i}=U\left(p^{k+1}-i p^{k}\right) \subseteq U\left(p^{k+1}\right)$ is the subgroup of $U\left(p^{k+1}\right)$ that fixes the first $i p^{k}$ standard basis vectors. We study the interaction of the two group actions by defining a left action of $\left(\Sigma_{i} \curlywedge \Delta_{k}\right) \times \Delta_{k+1}$ on $H_{i} \backslash U\left(p^{k}\right)$ by $(\phi, \delta) \cdot H_{i} g=H_{i} \phi g \delta^{-1}$. (Note that $\Sigma_{i} \prec \Delta_{k}$ commutes with $H_{i}$.) We look at how $F_{i}$ is built up according to the equivariant cells of this action, and we show that each piece is $(-1)-$ connected.

Let $I_{g} \subseteq\left(\Sigma_{i} \succ \Delta_{k}\right) \times \Delta_{k+1}$ be the isotropy group of $H_{i} g$. We need to establish $(-1)$-connectedness for

$$
\left[\left(\Sigma^{-1} \operatorname{Cof}_{i, d} \wedge S^{-i p^{k}}\right) \wedge\left(S^{p^{k+1}} \wedge \frac{\left(\Sigma_{i} \curlywedge \Delta_{k}\right) \times \Delta_{k+1}}{I_{g}}\right)_{\tilde{h} \Delta_{k+1}}\right]^{\tilde{h} \Sigma_{i} \iota_{k}} .
$$

when $1<i \leq p$. We know that $I_{g}$ injects into $\Delta_{k+1}$ (Lemma 5.1), so this can be reduced to

$$
\left[\left(\Sigma^{-1} \operatorname{Cof}_{i, d} \wedge S^{-i p^{k}}\right) \wedge\left(\Sigma_{i} \curlywedge \Delta_{k_{+}} \wedge S^{p^{k+1}}\right)_{\tilde{h} I_{g}}\right]^{\tilde{h} \Sigma_{i} \Delta_{k}}
$$

Using Lemma 6.6 we see that if $i>1$ then (6-5) can be rewritten as

$$
\left[\Sigma^{-1} \operatorname{Cof}_{i, d} \wedge S^{-i p^{k}} \wedge S^{p^{k+1}}\right]^{\tilde{h} I_{g}} .
$$

Here $I_{g}$ acts on $S^{-i p^{k}}$ through $\Sigma_{i} \prec \Delta_{k}$, and acts on $S^{p^{k+1}}$ through $\Delta_{k+1}$. We need to show that these "cancel out," so that we have a finite, $(-1)$-connected complex 
inside the homotopy fixed point set and can apply the Segal Conjecture to obtain our desired result.

Since $I_{g}$ is acting on $S^{p^{k+1}}$ through $\Delta_{k+1}$, which itself is acting on $S^{p^{k+1}}$ by the onepoint compactification of the regular representation, we know that $I_{g}$ acts on $S^{p^{k+1}}$ by the one-point compactification of a sum of copies of the regular representation of $I_{g}$. To finish the proof of the theorem, we need to show that the action of $I_{g}$ on $S^{i p^{k}}$ through $\Sigma_{i}>\Delta_{k}$ is also the one-point compactification of a sum of copies of the regular representation of $I_{g}$, even though $\Sigma_{i} \curlywedge \Delta_{k}$ is acting on $S^{i p^{k}}$ by the wreath product representation rather than by the regular representation of $\Sigma_{i} \Delta_{k}$. This is the content of Proposition 5.2.

Therefore the $I_{g}$-equivariant cells of $\left[\Sigma^{-1} \operatorname{Cof}_{i, d} \wedge S^{-i p^{k}} \wedge S^{p^{k+1}}\right]$ start no lower than the $I_{g}$-equivariant cells of $\Sigma^{-1} \operatorname{Cof}_{i, d}$, that is, in dimension 0 . Application of the Segal Conjecture then concludes the proof.

Remark 6.8 Theorem 6.3, as well as Corollary 6.2 have analogues at $p=2$, in which $S^{1} \wedge S^{V}$ is replaced with $S^{W}$, where $W$ is a real vector space. The proof of the theorem in the case $p=2$ is considerably easier than in the odd-primary case, and so we omit the details. There are two main reasons for the simpler proof when $p=2$.

(1) We do not have to restrict ourselves to odd spheres, and so instead of precomposing the identity functor from spaces to spaces with the functor $V \mapsto S^{1} \wedge S^{V}$ for a complex vector space $V$ (in order to obtain an odd-dimensional sphere), we can precompose with $W \mapsto S^{W}$ for a real vector space $W$. The resulting coefficient spectrum is simpler.

(2) The analysis of $F_{i}$ at odd primes is complicated considerably by the fact that the action of $\Delta_{k+1}$ on $S^{p^{k+1}}$ does not extend to an action of $U\left(p^{k}\right)$. Because of this, we can not make the often customary identification of spaces with an action of $U\left(p^{k}\right)$

$$
\begin{aligned}
S^{p^{k+1}} \wedge \operatorname{mor}_{\mathcal{J}}\left(\mathbb{C}^{i p^{k}}, \mathbb{C}^{p^{k+1}}\right) & \cong S^{p^{k+1}} \wedge\left(H_{i} \backslash U\left(p^{k+1}\right)\right)_{+} \\
& \cong S^{p^{k+1}} \wedge_{H_{i}} U\left(p^{k+1}\right)_{+}
\end{aligned}
$$

for the last space above is not even well-defined. However, the term $S^{p^{k+1}}$ came from the $S^{1}$ in $V \mapsto S^{1} \wedge S^{V}$. Thus this issue disappears entirely when $p=2$. 


\section{Delooping structure maps: the unitary case}

This section establishes a parallel result to Theorem 6.1, but for the $p$-completed Taylor tower of $V \mapsto B U(V)$ studied in work of Arone [2] and Arone-Lesh [4]. As discussed in those works, the Taylor tower for $V \mapsto B U(V)$ shares many interesting properties with the Taylor tower of the identity functor on pointed spaces. Not only are the layers nontrivial only at powers of $p$, but other analogies also hold; for example, the spectra associated to the layers are related to partition complexes, and they are wedge summands of suspension spectra of homotopy orbit spaces of actions on spheres. In [4], the authors conjecture an analogue of the Whitehead Conjecture involving the layers of the $p$-localized Taylor tower of $V \mapsto B U(V)$. The program proposed involves a delooping of the structure maps in the tower, and the goal of this section is to contribute to this circle of ideas by establishing that the structure map, which connects the $p^{k}$ th layer directly to the $p^{k+1}$ st layer, can be delooped $k-1$ times (at least after $p$-completion).

By analogy with the notation of Section 6, let $\operatorname{DU}_{k}(V)$ denote the $p^{k}$ th layer of the Taylor tower for $V \mapsto B U(V)$ completed at $p$ and evaluated at $V$. Our goal is to prove the following theorem.

Theorem 7.1 Let $k>0$. Let $\operatorname{DU}_{k}(V)$ be the $p^{k}$ th layer of the $p$-completed Weiss tower of the functor $V \mapsto B U(V)$ from complex vector spaces to spaces. Then the $(k-1)$-fold looping map

$$
\Omega^{k-1}: \operatorname{hoNat}\left(\mathrm{B}^{k-1} \mathrm{DU}_{k}, \mathrm{~B}^{k} \mathrm{DU}_{k+1}\right) \rightarrow \operatorname{hoNat}\left(\mathrm{DU}_{k}, \mathrm{~B} \mathrm{DU}_{k+1}\right)
$$

is surjective on $\pi_{0}$.

Corollary 7.2 Let $p$ be a prime and let $n=p^{k}$ for $k>0$. Let $\operatorname{DU}_{k}(V)$ be the $p^{k}$ th layer of the $p$-completed Weiss tower of $B U(-)$ evaluated at a finite-dimensional complex vector space $V$. Then the connecting map

$$
\kappa_{k}: \mathrm{DU}_{k}(V) \rightarrow \mathrm{B} \mathrm{DU}_{k+1}(V)
$$

admits a $(k-1)$-fold delooping in the homotopy category of functors.

The case $V=\mathbb{C}$ of the corollary was conjectured in [4].

The outline for the proof of Theorem 7.1 is largely the same as that of Section 6. However, the proof of the critical delooping result (Theorem 7.3, corresponding to Theorem 6.3) has different aspects, including an application of the Segal Conjecture for a nonfinite group, which is what explains the switch to $p$-completion. Thus in 
this section we only summarize the set-up corresponding to Section 6, but we give the proof of Theorem 7.3 in detail.

The prerequisite results we need come from [4] and [2]. In the analogy between the Taylor tower for the identity functor on pointed spaces and the Taylor tower for the functor $V \mapsto B U(V)$ on finite-dimensional complex vector spaces, the role of the transitive elementary abelian subgroup $\Delta_{k} \subseteq \Sigma_{p^{k}}$ is played by the projective elementary abelian $p$-subgroup $\Gamma_{k} \subseteq U\left(p^{k}\right)$. The role of $\mathrm{GL}_{k}\left(\mathbb{F}_{p}\right)$, the Weyl group of $\Delta_{k}$ in $\Sigma_{p^{k}}$, is played by the symplectic group $\operatorname{Sp}_{2 k}\left(\mathbb{F}_{p}\right)$, the Weyl group of $\Gamma_{k}$ in $U\left(p^{k}\right)$. There is a Steinberg idempotent $\epsilon_{k}$ in $\operatorname{Sp}_{2 k}\left(\mathbb{F}_{p}\right)$ playing the same role as the Steinberg idempotent in $\mathrm{GL}_{k}\left(\mathbb{F}_{p}\right)$.

Recall from Remark 3.5 that $L_{p}$ is the $p$-completion functor. It is proved in [4] that the infinite loop space $\mathrm{DU}_{k}(V)$ has the form

$$
\mathrm{DU}_{k}(V) \simeq \Omega^{k-1} \Omega^{\infty}\left[\epsilon_{k} \cdot L_{p} \Sigma^{\infty}\left(S^{V}\right)_{\tilde{h} \Gamma_{k}}^{\wedge p^{k}}\right]
$$

(See [4, Theorems 11.1 and 11.2].) If we now let $\kappa_{k}$ be the natural transformation of functors from finite-dimensional complex vector spaces to pointed spaces that is the structure map $\mathrm{DU}_{k}(V) \rightarrow B \mathrm{DU}_{k+1}(V)$, we have the situation described in the following diagram:

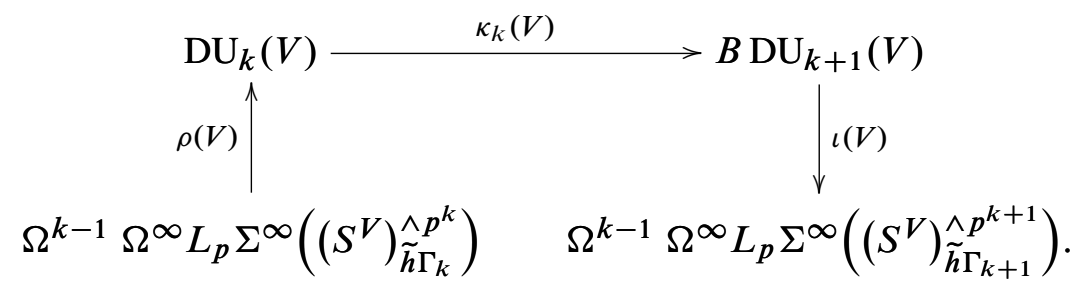

The following theorem is the fundamental delooping result for the unitary case, corresponding to Theorem 6.3 for the homotopy case.

Theorem 7.3 For any nonnegative integer $d$, the $d$-fold looping map

$$
\begin{gathered}
\operatorname{hoNat}\left[\Omega^{\infty} L_{p} \Sigma^{\infty}\left(S^{p^{k} V}\right)_{\tilde{h} \Gamma_{k}}, \quad \Omega^{\infty} L_{p} \Sigma^{\infty}\left(S^{p^{k+1} V}\right)_{\tilde{h} \Gamma_{k+1}}\right] \\
\downarrow \\
\operatorname{hoNat}\left[\Omega^{d} \Omega^{\infty} L_{p} \Sigma^{\infty}\left(S^{p^{k} V}\right)_{\tilde{h} \Gamma_{k}}, \quad \Omega^{d} \Omega^{\infty} L_{p} \Sigma^{\infty}\left(S^{p^{k+1} V}\right)_{\tilde{h} \Gamma_{k+1}}\right]
\end{gathered}
$$

is an epimorphism on $\pi_{0}$.

Algebraic 83 Geometric Topology, Volume 8 (2008) 
To set up the proof of Theorem 7.3, we follow Section 6 and rearrange the target of (7-3) using adjointness to the form

(7-4) $\operatorname{hoNat}_{V}\left[S^{d} \wedge \Sigma^{\infty} \Omega^{\infty}\left(L_{p} S^{-d} \wedge \Sigma^{\infty} S^{p^{k} V}\right)_{\tilde{h} \Gamma_{k}}, \quad \Sigma^{\infty}\left(L_{p} S^{p^{k+1} V}\right)_{\tilde{h} \Gamma_{k+1}}\right]$.

The target of (7-4) is the $p$-completion of a homogeneous functor of degree $p^{k+1}$, whose representing spectrum is $\Sigma^{\infty}\left(U\left(p^{k+1}\right) / \Gamma_{k+1}\right)_{+}$. In particular, the target is $p^{k+1}$-excisive. For the source, we have the following analogue of Lemma 6.7; note that arguments from Remark 3.5 show that the natural transformation space in (7-4) is unaffected if the $p$-completion is removed from the source.

Lemma 7.4 The functor

$$
S^{d} \wedge \Sigma^{\infty} \Omega^{\infty}\left(S^{-d} \wedge \Sigma^{\infty} S^{p^{k} V}\right) \widetilde{h} \Gamma_{k}
$$

has nontrivial homogeneous layers only in dimensions that are multiples of $p^{k}$. The $i p^{k}$ th layer is represented by the spectrum

$$
\Sigma^{\infty} U\left(i p^{k}\right)_{+} \wedge \Sigma_{i} 2 \Gamma_{k} S^{-(i-1) d}
$$

where $\Gamma_{k}$ acts trivially on $S^{-(i-1) d}$ and $\Sigma_{i}$ acts on $S^{-(i-1) d}$ by the dual of the one-point compactification of $d$ copies of the reduced standard representation.

Proof of Theorem 7.3 In this proof the spectra involved are $p$-completed. Following the same reasoning as in the proof of Theorem 6.3 (but with the help of Remark 3.5 when it comes to computing natural transformations into $p$-completed functors) we need to show that for $1<i \leq p$,

$$
\left[\Sigma^{-1} \operatorname{Cof}_{i, d} \wedge\left(H_{i} \backslash U\left(p^{k+1}\right)_{+}\right)_{\tilde{h} \Gamma_{k+1}}\right]^{\tilde{h} \Sigma_{i} \lambda \Gamma_{k}}
$$

is $(-1)$-connected. If $I_{g}$ is the isotropy group of $H_{i} g$ under the action of $\Sigma_{i} \prec \Gamma_{k} \times$ $\Gamma_{k+1}$, then Lemma 6.6 tells us that (7-5) is built by cofibrations from spectra of the form

$$
\begin{aligned}
{\left[\Sigma^{-1} \operatorname{Cof}_{i, d} \wedge\left(\frac{\Sigma_{i}\left\langle\Gamma_{k} \times \Gamma_{k+1}\right.}{I_{g}+}\right)_{\tilde{h} \Gamma_{k+1}}\right]^{\left.\tilde{h} \Sigma_{i}\right\rangle \Gamma_{k}} } & \simeq\left[\Sigma^{-1} \operatorname{Cof}_{i, d} \wedge\left(\Sigma_{i} \lambda \Gamma_{k+}\right)_{\tilde{h} I_{g}}\right]^{\tilde{h} \Sigma_{i}\left\langle\Gamma_{k}\right.} \\
& \simeq\left[\Sigma_{i} 2 \Gamma_{k+} \wedge\left(\Sigma^{-1} \operatorname{Cof}_{i, d}\right)_{\tilde{h} I_{g}}\right]^{\tilde{h} \Sigma_{i} \lambda \Gamma_{k}} \\
& \left.\simeq\left[\operatorname{Map}\left(\Sigma_{i}\right\rangle \Gamma_{k+,}, \Sigma^{-1} \operatorname{Cof}_{i, d}\right)^{\tilde{h} I_{g}}\right]^{\tilde{h} \Sigma_{i} \lambda \Gamma_{k}} \\
& \simeq\left[\Sigma^{-1} \operatorname{Cof}_{i, d}\right]^{\tilde{h} I_{g}}
\end{aligned}
$$

Algebraic 83 Geometric Topology, Volume 8 (2008) 
To compute this last homotopy fixed point set, we need to know a little more about the isotropy subgroup $I_{g}$ as a subgroup of $\Sigma_{i} \prec \Gamma_{k}$. Recall that $I_{g}=\{(\phi, \gamma) \in$ $\left(\Sigma_{i} \prec \Gamma_{k}\right) \times \Gamma_{k+1} \mid \exists h \in H_{i}$ s.t. $\left.g \gamma g^{-1}=\phi h\right\}$. We can think of $I_{g}$ being embedded in $U\left(p^{k+1}\right)$ as $\left(g \Gamma_{k+1} g^{-1}\right) \cap\left(\Sigma_{i} 2 \Gamma_{k} \times H_{i}\right)$. By inspection of the intersection, the central $S^{1}$ of $\Gamma_{k+1}$ is contained in $I_{g}$, and since $I_{g} \hookrightarrow \Gamma_{k+1}$, we know that $I_{g}$ is an extension of $S^{1}$ by an elementary abelian $p$-group $E$.

The subgroup $I_{g}$ acts on $\operatorname{Cof}_{i, d}$ by first projecting to $\Sigma_{i} 2 \Gamma_{k}$, then projecting to $\Sigma_{i}$, and then acting through the action of $\Sigma_{i}$ on $\operatorname{Cof}_{i, d}$. Thus $\operatorname{Cof}_{i, d}$ has a finite number of cells as an $I_{g}$-space (because it has a finite number of cells as a $\Sigma_{i}$-space), and each cell has the form $I_{g} / K \times$ disk where $I_{g} / K=*$ if $i<p$, or possibly $I_{g} / K \cong \mathbb{Z} / p$ if $i=p$.

Recall that $I_{g}$ is an extension of the central circle $S^{1}$ by an elementary abelian $p$-group $E$. We define corresponding subgroups $I_{g}^{\prime}$ and $\left(I_{g}^{\prime}\right)_{n}$ as follows:

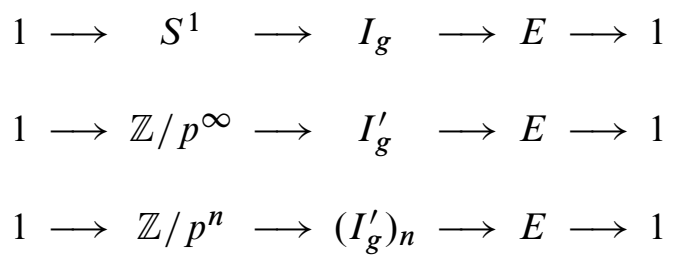

Recall that we have $p$-completed $\operatorname{Cof}_{i, d}$. The map $B I_{g}^{\prime} \rightarrow B I_{g}$ is an $H \mathbb{Z} / p_{*}-$ isomorphism, and therefore so is

$$
\left[\operatorname{Cof}_{i, d}\right]^{\tilde{h} I_{g}} \rightarrow\left[\operatorname{Cof}_{i, d}\right]^{\tilde{h} I_{g}^{\prime}}
$$

for example by looking at the Bousfield-Kan spectral sequence for the homotopy groups of an inverse limit.

We assert that the natural maps $\left[\mathrm{Cof}_{i, d}\right]^{\tilde{h} I_{g}^{\prime}} \rightarrow\left[\mathrm{Cof}_{i, d}\right]^{\widetilde{h}\left(I_{g}^{\prime}\right)_{n}}$ induce a map

$$
\left[\operatorname{Cof}_{i, d}\right]^{\tilde{h} I_{g}^{\prime}} \rightarrow \underset{n}{\operatorname{holim}}\left[\operatorname{Cof}_{i, d}\right]^{\tilde{h}\left(I_{g}^{\prime}\right)_{n}}
$$

that is an $H \mathbb{Z} / p_{*}$-isomorphism. This is because

$$
\left[\operatorname{Cof}_{i, d}\right]^{\widetilde{h}\left(I_{g}^{\prime}\right)_{n}} \simeq\left(\left[\operatorname{Cof}_{i, d}\right]^{\tilde{h} \mathbb{Z} / p^{n}}\right)^{\tilde{h} E} \simeq\left(\operatorname{Map}\left(B \mathbb{Z} / p^{n}, \operatorname{Cof}_{i, d}\right)\right)^{\tilde{h} E}
$$


and so

$$
\begin{aligned}
\operatorname{holim}_{n}\left[\operatorname{Cof}_{i, d}\right]^{\tilde{h}\left(I_{g}^{\prime}\right)_{n}} & \simeq \underset{n}{\operatorname{holim}}\left[\operatorname{Map}\left(B \mathbb{Z} / p^{n}, \operatorname{Cof}_{i, d}\right)\right]^{\tilde{h} E} \\
& \simeq\left[\operatorname{Map}\left(\operatorname{hocolim} B \mathbb{Z} / p^{n}, \operatorname{Cof}_{i, d}\right)\right]^{\tilde{h} E} \\
& \simeq\left[\operatorname{Map}\left(B \mathbb{Z} / p^{\infty}, \operatorname{Cof}_{i, d}\right)\right]^{\tilde{h} E} \\
& \simeq\left(\left[\operatorname{Cof}_{i, d}\right]^{\tilde{h} \mathbb{Z} / p^{\infty}}\right)^{\tilde{h} E} \\
& \simeq\left[\operatorname{Cof}_{i, d}\right]^{\tilde{h} I_{g}^{\prime}}
\end{aligned}
$$

We need to show that the right-hand side of (7-6) is 0-connected. By the Segal Conjecture for finite groups, since $\operatorname{Cof}_{i, d}$ is finite and has its first $\Sigma_{i}$-cell in dimension 1 , we know that $\pi_{0}\left[\operatorname{Cof}_{i, d}\right]^{\widetilde{h}\left(I_{g}^{\prime}\right) n}=0$ for all $n$. Using the short exact sequence for the homotopy groups of the homotopy inverse limit of a tower, we are done once we show that $\lim _{n}^{1} \pi_{1}\left[\operatorname{Cof}_{i, d}\right]^{\widetilde{h}\left(I_{g}^{\prime}\right) n}=0$. However, the homotopy groups of $\left[\operatorname{Cof}_{i, d}\right]^{\widetilde{h}\left(I_{g}^{\prime}\right) n}$ are finitely generated over the $p$-adic integers, and this is enough to imply triviality of $\lim ^{1}$ because such groups are compact.

This finishes the proof of Theorem 7.3.

\section{References}

[1] S T Ahearn, N J Kuhn, Product and other fine structure in polynomial resolutions of mapping spaces, Algebr. Geom. Topol. 2 (2002) 591-647 MR1917068

[2] G Arone, The Weiss derivatives of $B \mathrm{O}(-)$ and $B \mathrm{U}(-)$, Topology 41 (2002) 451-481 MR1910037

[3] G Z Arone, W G Dwyer, Partition complexes, Tits buildings and symmetric products, Proc. London Math. Soc. (3) 82 (2001) 229-256 MR1794263

[4] G Arone, K Lesh, Filtered spectra arising from permutative categories, J. Reine Angew. Math. 604 (2007) 73-136 MR2320314

[5] G Arone, M Mahowald, The Goodwillie tower of the identity functor and the unstable periodic homotopy of spheres, Invent. Math. 135 (1999) 743-788 MR1669268

[6] A K Bousfield, $K$-localizations and $K$-equivalences of infinite loop spaces, Proc. London Math. Soc. (3) 44 (1982) 291-311 MR647434

[7] E D Farjoun, Cellular spaces, null spaces and homotopy localization, Lecture Notes in Mathematics 1622, Springer, Berlin (1996) MR1392221

[8] M Hovey, Model categories, Mathematical Surveys and Monographs 63, American Mathematical Society, Providence, RI (1999) MR1650134 
[9] N J Kuhn, A Kahn-Priddy sequence and a conjecture of $G W$ Whitehead, Math. Proc. Cambridge Philos. Soc. 92 (1982) 467-483 MR677471

[10] N J Kuhn, S B Priddy, The transfer and Whitehead's conjecture, Math. Proc. Cambridge Philos. Soc. 98 (1985) 459-480 MR803606

[11] M A Mandell, J P May, S Schwede, B Shipley, Model categories of diagram spectra, Proc. London Math. Soc. (3) 82 (2001) 441-512 MR1806878

[12] S Schwede, B Shipley, Equivalences of monoidal model categories, Algebr. Geom. Topol. 3 (2003) 287-334 MR1997322

[13] M Weiss, Orthogonal calculus, Trans. Amer. Math. Soc. 347 (1995) 3743-3796 MR1321590

Kerchof Hall, University of Virginia

PO Box 400137, Charlottesville VA 22904, USA

Department of Mathematics, University of Notre Dame

Notre Dame IN 46556, USA

Department of Mathematics, Union College

Schenectady NY 12309, USA

zga2m@virginia.edu, dwyer.1@nd.edu, leshk@union.edu

http://www.math.virginia.edu/ zga2m/, http://www.nd.edu/ wgd/, http://www . math. union.edu/ leshk/

Received: 24 April 2007 Revised: 30 November 2007 